\title{
Peptide therapeutics and assemblies for cancer immunotherapy
}

\author{
Mingming Li, Xinran Zhao, Jianfang Dai and Zhilin Yu*
}

\begin{abstract}
Immunotherapy has been considered as one of the most promising strategies for protection against cancer cells due to the tremendous advantages arising from host immune defense. However, establishing versatile strategies with high biosafety and the capability for efficient modulation of immune responses remains challenging. The structural features resembling native proteins of peptides bestow their great potential to address these challenges via either directly eliciting immune responses or improving the efficacy of therapeutics. This review summarizes the progress of cancer immunotherapy achieved based on the strategies utilizing short peptides as therapeutic agents or peptide assemblies as delivery scaffolds, beyond long sequences like proteins and polypeptides. Starting from a brief introduction of cancer immunotherapy, we outline the peptide sequences in terms of their specific functions including immune checkpoint blockades, vaccine antigens and adjuvants. We particularly highlight peptide-based nanomaterials as scaffolds for targeting delivery or co-delivery of multiple therapeutics to enhance immunogenicity. The extraordinary therapeutic efficacy of the limited examples covered here demonstrates the great potency of the peptide-based strategies in modulating immune responses, thus potentially facilitating the clinical translation of cancer immunotherapy in the future.
\end{abstract}

Keywords: cancer immunotherapy, peptides, self-assembly, checkpoint blockades, combinatorial immunotherapy

\section{INTRODUCTION}

Cancers are one of main life-threatening diseases and their therapy suffers from the challenges in sufficient treatments, despite the progress made in conventional strategies including surgical resection, chemotherapy, and radiation therapy $[1,2]$. As an alternative approach, im- munotherapy has attracted broad attention over the past few decades due to its advantages in curative efficacy and lowered side effect arising from drug off-target [3,4]. In contrast to directly attack cancer cells, immunotherapy elicits host natural immune responses and thereby killing cancer cells. Since the first marketed immunotherapy for hairy cell leukaemia, a variety of cancer vaccines have been developed and applied in cancer immunotherapy [5]. Recently, the breakthrough of cancer immunotherapy has been achieved based on the new strategies provoking immune responses via targeting different immune cells [6]. In principle, the immune system consists of innate immune system and adaptive immune systems dependent on the involved immune cells, including microphages, monocytes, neutrophils, and dendritic cells in innate immune system, and $\mathrm{T}$ or $\mathrm{B}$ lymphocytes in adaptive immune system. On the basis of the mechanism for immune activation, therapeutic agents targeting different immune responses could be classified into cancer vaccines, immune adjuvants, cytokines, checkpoint blockades, and engineered $\mathrm{T}$ cells, among other emerging categories. Currently checkpoint blockade immunotherapy $[7,8]$ and adoptive immunotherapy using engineering T-cells [9] are two promising strategies used in clinical trials. The checkpoints of the programmed cell death 1 (PD-1) and its ligand PD-L1 or cytotoxic T lymphocytes antigen 4 (CTLA-4) are two conventional targeting sites for the blockades including antibodies or small molecular drugs. Adoptive transfer of engineered $\mathrm{T}$ cells like chimeric antigen receptors $\mathrm{T}(\mathrm{CAR} \mathrm{T})$ cells to replace natural $\mathrm{T}$ cell receptors also allows for directly eliciting immune responses [10-13]. Despite the great curative potential, the failure of many clinical trials arising from low immunogenicity and serious adverse side

Key Laboratory of Functional Polymer Materials, Ministry of Education, State Key Laboratory of Medicinal Chemical Biology, Institute of Polymer Chemistry, College of Chemistry Nankai University, Tianjin 300071, China

* Corresponding author (email: yzh026@nankai.edu.cn) 
effect such as cytokine release syndrome significantly hampers its further development. Hence, new therapeutic agents for efficiently modulating immune responses and novel delivery scaffolds for reducing adverse effect such as autoimmune side effect are demanded.

Peptides, consisting of amino acids analogue to native proteins, have been broadly utilized in development of drugs and biomaterials for tissue regeneration and drug delivery [14-16]. Due to the potential capability derived from native proteins or mimicking structural features of protein substrates, peptide sequences might exhibit the propensity to associate with foreign pathogens or cancer cells and serves as peptide therapeutics. For examples, inspired by the innate immune responses arising from antimicrobial peptides, antimicrobial agents have been developed by rational design of peptide sequences with broad spectrum antibiotics to defend bacteria and fungi $[17,18]$. Compared to large protein antibodies, short peptide therapeutics exhibit several remarkable advantages in administration and tumor accumulation. The shortened sequences of peptides are readily synthesized and also potentially benefit the penetration into solid tumor tissues. The structure of short peptides can also be precisely tuned to prevent any allergies or autoimmune reactions arising from drug contaminants. The therapeutic efficacy of short peptides does not require stable global conformational analogue to protein antibodies, which is critical for the curative efficacy of antibodies and leads to challenges in administration of protein antibodies. In addition, modulating the noncovalent interactions of peptide therapeutics allows for promotion of peptide self-assembly into nanomedicines, which usually show controllable pharmacokinetics. On the other hand, nanostructures formed by peptides possess specific features when serving as delivery platforms compared to polymeric systems. While the natural component of peptides renders their excellent cytocompatibility, rational design of peptides allows for precisely tailoring their association, thus creating nanostructures with controllable morphologies and subsequently developing functional biomaterials for disease diagnosis and therapy $[19,20]$. Peptide assemblies responsive to tumor microenvironment, particularly responsive to the biomarkers, are ideal scaffolds for efficient tumor imaging [21-23] and targeting delivery $[24,25]$ and have been broadly utilized in conventional cancer therapy [26-28]. Combining these advantages with the potential transmembrane capability, peptides possess great potential in cancer immunotherapy serving as either therapeutics or delivery scaffolds [29-31].
Thus far, synthetic short peptides and their assemblies have been employed in different classes of cancer immunotherapy (Fig. 1) [32,33]. Peptide epitopes derived from the domains within native proteins are potentially capable of interacting with receptors present in innate or adaptive immune cells or cancer cells, thus endowing their therapeutic functions to modulate host immune system [34,35]. In addition, molecular evolution technology allows for de novo design of peptides targeting the receptors participating in suppression or activation of immune system, leading to an alternative approach to discovery of peptide immune therapeutics. Thus far, a considerable number of short peptides serving as the checkpoint blockades [36,37], cancer vaccines $[38,39]$ and adjuvants [40] have been developed. On the other hand, peptide assemblies have been broadly utilized as nanocarriers in targeting delivery of immune therapeutic agents ranging from large objects like cells and antibodies to small drugs into tumor sites [41,42]. Based on the morphological transition of peptide assemblies, peptide nanocarriers can also increase the circulation time of therapeutics [43-45]. Recently, peptide assemblies have attracted specific attention in the combinatory conventional therapeutic and immunotherapy approaches [4650], due to their versatility for co-loading multiple cargoes. Despite the great potential of peptides in cancer immunotherapy, the progress of peptide-based strategies has not been summarized yet.

This review summarizes the strategies of peptide-based

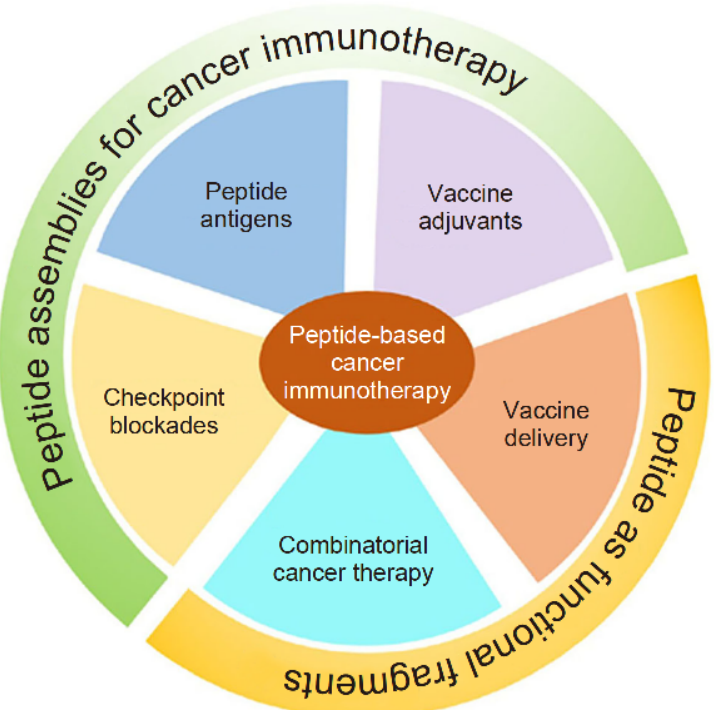

Figure 1 Applications of peptide epitopes or peptide assemblies in cancer immunotherapy ranging from directly serving as therapeutics or as delivery systems for therapeutics. 
cancer immunotherapy in terms of the functions of peptides in therapeutic processes. We initially outline peptide-based immunotherapy utilizing peptides as immune therapeutics. Subsequently, the applications of peptide nanostructures as delivery scaffolds for therapeutic agents will be introduced. We particularly highlight the synergistic immunotherapy or the combinatorial immunotherapy involving conventional chemotherapy and photodynamic therapy. It is worth noting that this review only covers the cancer immunotherapy utilizing short peptides as therapeutics or delivery scaffolds, beyond those strategies involving long sequences like proteins and polypeptides. In addition, immunotherapeutic peptides have emerged a couple of decades ago and a considerable number of therapeutic peptides for distinct modalities and cancers have been developed thus far. To precisely illustrate the covered references, the immunotherapeutic peptides summarized in this manuscript are confined as those mostly discovered within the past decade, and also the peptide delivery platforms are created recently for the burgeoning immunotherapeutic modalities such as checkpoint blockades and combinatorial immunotherapy. On the basis of the sophisticated properties of peptides, summarizing the strategies of peptide-based cancer immunotherapy allows for overviewing the current status of the applications of peptides in immunotherapy and potentially stimulating the development of new strategies to improve the curative efficacy, thus potentially facilitating the clinical translation of cancer immunotherapy in the future.

\section{PEPTIDE CHECKPOINT BLOCKADES}

Immune checkpoints are referred to as the negative regulators present in the immune system to maintain homeostasis and prevent autoimmunity from attacking cells indiscriminately [51]. However, immune checkpoint mechanisms can be also activated in cancer cells to inhibit the nascent antitumor immune responses and thus leading to the escape and growth of cancer cells [52,53]. Inhibition of the immune checkpoints allows for blocking the immune evasion of cancer cells and stimulation of the activity of the immune cells such as cytotoxic $\mathrm{T}$ cells to protect against cancer cells, which has been considered as a promising and effective strategy for cancer immunotherapy [54]. In principle, the primary inhibitory receptors expressed by activated $\mathrm{T}$ cells include $\mathrm{PD}-1$ [55], CTLA-4 [56], lymphocyte-activation gene 3 (LAG-3) [57-59], T-cell immunoglobulin 3 (TIM-3) [6062 , and T cell-immunoglobulin and ITIM domain (TIGIT) [63-65]. Hence these receptors serving as targeting immune checkpoints allow for development of inhibitors for activating immune responses, as represented by PD-1 and CTLA-4 broadly used in current preclinical studies and clinical trials (Fig. 2) [63].

CTLA-4 is a transmembrane glycoprotein highly expressed on regulatory or activated $\mathrm{T}$ cells [66]. CTLA-4 exhibits a high degree of homology with the costimulatory molecule receptor (CD28) on the surface of T cells and enables to bind with $\mathrm{B} 7$ proteins, i.e. CD80 (B7-1) and CD 86 (B7-2), with an associating affinity approximately 20-fold greater than CD 28 (Fig. 2). This allows CTLA-4 to outcompete CD28 for B7 binding and thus preventing release of CD28-B7 costimulatory signals and inhibiting $\mathrm{T}$ cell activation [67-69]. Current research indicates that CTLA-4 inhibits T cell immune responses potentially through either signaling or non-signaling pathways. The signaling pathway suggests that CTLA-4 activates the phosphatases to dephosphorylate the signals for $\mathrm{T}$ cell receptors (TCR). The non-signaling pathway indicates that CTLA-4 potentially captures and removes CD80 and CD86 proteins from the membrane of antigen presenting cells (APC) through the transendocytosis process, thus attenuating CD28 activation [70,71]. PD-1 is another immune checkpoint belonging to the extended B7/CD28 family and highly expressed in activated T cells, $B$ cells, natural killer cells, dendritic cells, and tumorassociated macrophages [72]. PD-1 protein consists of an extracellular domain, a transmembrane domain, and an intracellular domain. In contrast to CTLA-4 affecting naïve T-cells, PD-1 is conventionally expressed on mature $\mathrm{T}$ cells and regulates effector $\mathrm{T}$ cell activity within the tumor microenvironment (Fig. 2) [73]. The ligands for PD-1 including PD-L1 (B7-H1 or CD 274) and PD-L2 (B7-DC or CD 273) are expressed by APCs and tumor cells $[74,75]$. Binding PD-1 with its ligands leads to inactivation of $\mathrm{T}$ cell kinase and dephosphorylation of TCR signals, thus ultimately reducing production of inflammatory cytokines and regulating $\mathrm{T}$ cell activity $[76,77]$. Therefore, blocking the PD-1/PD-L1 pathway maintains the activity of tumor-specific $\mathrm{T}$ cells and allows the immune system to re-identify and attack tumor cells, thereby preventing immune evasion of tumor cells.

Thus far, while the common CTLA-4 checkpoint inhibitors are antibodies, the explicit sequences of PD-1 and PD-L1 and their complex structures inspire rational design of short biomimetic peptides with the capability to outcompete the PD-1 and PD-L1 association (Table 1). Despite the achievement of monoclonal antibodies as PD1/PD-L1 checkpoint inhibitors in clinical trials, the problems of antibodies including poor penetrance through 


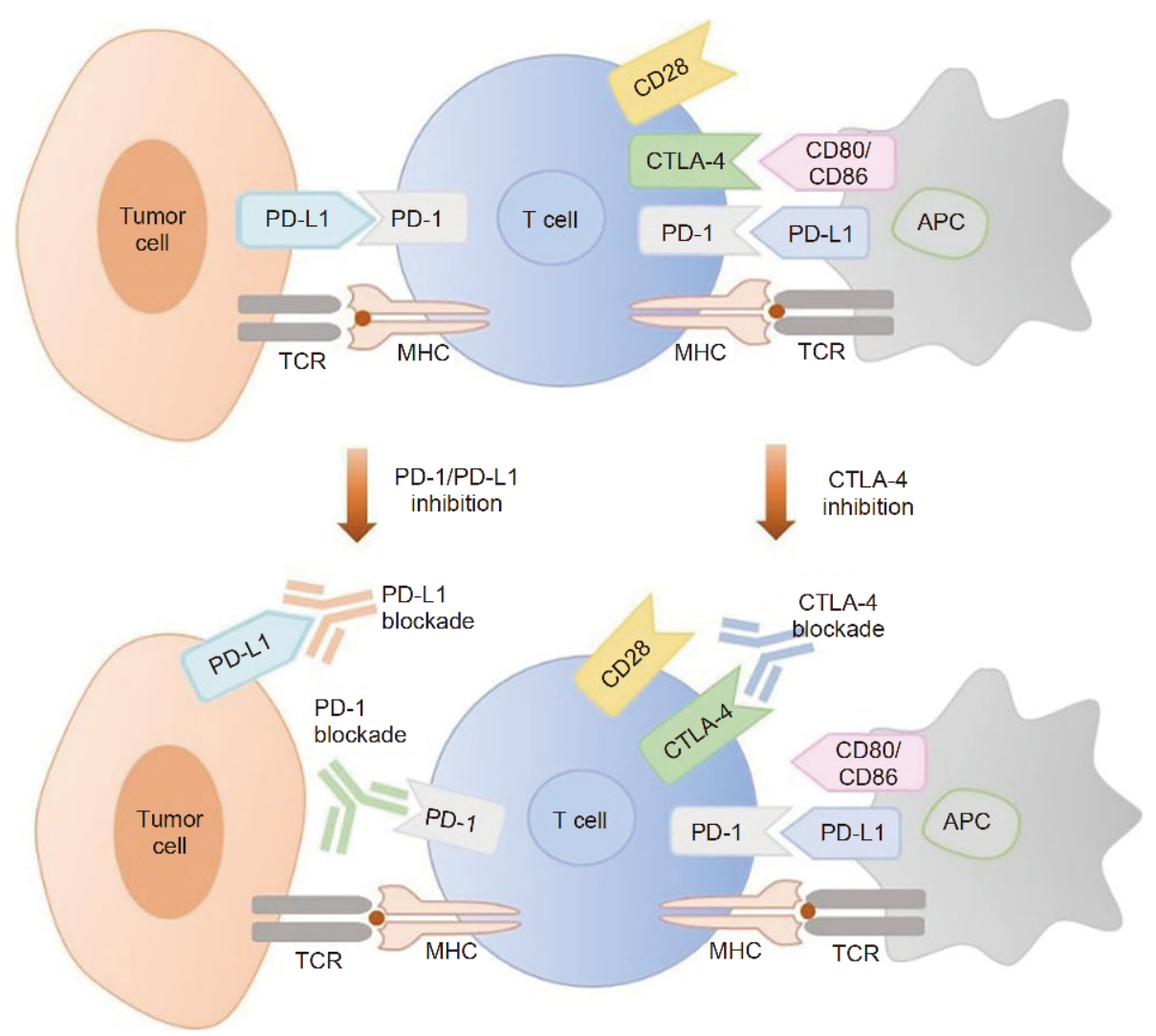

Figure 2 Schematic illustration of the mechanism of immunotherapy based on inhibition of either the cytotoxic T-lymphocyte-associated antigen 4 (CTLA-4)-mediated immune checkpoint or the programmed cell death protein 1 (PD-1) and its ligand PD-L1 checkpoint.

solid-tumor tissues, low stability, high production cost, limited administration approaches, and less controllable pharmacokinetics significantly hamper the clinical translation [78,79]. In addition, antibody inhibitors show limited capability to activate cytotoxic immune responses through natural killer cells and macrophages. Peptides or other small organic molecules serving as the PD-1/PD-L1 checkpoint blockades are alternative drugs to address these problems of monoclonal antibodies. Considering the extraordinary biocompatibility and easy synthesis of short peptides, peptide-based PD-1/PD-L1 inhibitors have attracted broad attention in cancer immunotherapy. This section primarily covers the development of peptide PD-1/PD-L1 inhibitors and summarizes the available sequences in both clinical and preclinical trials.

One of the pioneering peptide PD-1/PD-L1 inhibitors is AUNP-12 that was discovered by Aurigene Discovery Technologies and Laboratories Pierre Fabre with a pharmacokinetic advantage compared to antibody inhibitors, on the basis of the non-linear combination of the 7- to 30-mer domains within human and murine PD-1 extracellular domain [80]. In preclinical studies, AUNP12 showed the remarkable capability in inhibition of growth of multiple tumors including B16F10 mouse melanoma cells and mouse breast $4 \mathrm{~T} 1$ cancer cells. In vivo studies demonstrated that mediating interferon- $\gamma$ (IFN- $\gamma)$ production is the potential signal pathway for AUNP-12 inhibitory activity. The studies of the structure-activity relationship of AUNP-12 revealed that either deletion of the C-terminal eight residues or acylation of the $\mathrm{N}$ terminal serine residues led to loss of the inhibitory activity, whereas the activity was retained by removal of the branched domain or acylation of the C-terminal lysine residue. A short peptidomimetic compound of AUNP-12 peptide was also discovered and exhibits an even better activity in cancer immunotherapy compared to AUNP12. Following this study, a series of peptidomimetic PD-1/ PD-L1 inhibitors have been developed by Aurigene based on this concept.

In addition, Chang et al. [81] developed the first proteolysis-resistant D-peptide PD-1/PD-L1 interaction antagonist ( ${ }^{\mathrm{D} P A-1}$, NYSKPTDRQYHF) on the basis of 
Table 1 Peptide therapeutics in cancer immunotherapy

\begin{tabular}{|c|c|c|c|c|}
\hline Therapeutics & Name & Peptide sequences & Pathway & Ref. \\
\hline \multirow{10}{*}{ PD-1/PD-L1 blockades } & AUNP-12 & $\begin{array}{l}(\mathrm{SNTSESF})_{2} \mathrm{KFRVTQ} \\
\text {-LAPKQIKE-NH } \\
\end{array}$ & PD-1 & {$[80]$} \\
\hline & ${ }^{\mathrm{D}} \mathrm{PPA}-1$ & NYSKPTDRQYHF & PD-L1 & {$[81]$} \\
\hline & ${ }^{\mathrm{D}} \mathrm{PPA}-2$ & KHAHHTHNLRLP & PD-L1 & {$[81]$} \\
\hline & HAC-I & HVIHEGTVVI & PD-L1 & {$[82]$} \\
\hline & HAC-V & HVVHEGTVVI & PD-L1 & {$[82]$} \\
\hline & TPP-1 & $\begin{array}{l}\text { SGQYASYHCWC } \\
\text {-WRDPGRSGGSK }\end{array}$ & PD-L1 & {$[83]$} \\
\hline & PDLong1 & $\begin{array}{l}\text { FMTYWHLLN } \\
\text {-AFTVTVPKDL }\end{array}$ & PD-L1 & {$[84]$} \\
\hline & Peptide-57 & $\begin{array}{c}\text { Cyclic }[\mathrm{F}(\mathrm{NMe}) \mathrm{ANPHLSWSW} \\
(\mathrm{NMe})[\mathrm{NLe}](\mathrm{NMe})[\mathrm{NLe}] \mathrm{R}(\mathrm{Scc})] \mathrm{G}\end{array}$ & PD-L1 & {$[85]$} \\
\hline & Peptide-71 & $\begin{array}{l}\text { Cyclic }[\mathrm{F}(\mathrm{NMe}) \mathrm{F}(\mathrm{NMe})[\mathrm{NLe}](\text { Sar }) \\
\text { DV(NMe)FY(Sar)WYL(Scc)]G }\end{array}$ & PD-L1 & {$[85]$} \\
\hline & Peptide-99 & Cyclic[FLIVIRDRVFR(Scc)]G & PD-L1 & {$[85]$} \\
\hline \multirow{21}{*}{ Peptide antigens } & $\mathrm{OVA}_{257-264}$ & SIINFEKL & $\mathrm{CD}^{+} \mathrm{T}$ cell & {$[86]$} \\
\hline & $\mathrm{OVA}_{253-266}$ & EQLESIINFEKLTE & $\mathrm{CD}^{+} \mathrm{T}$ cell & {$[87]$} \\
\hline & $\mathrm{OVA}_{323-339}$ & $\begin{array}{l}\text { ISQAVHAA } \\
\text {-HAEINEAGR }\end{array}$ & $\mathrm{CD}^{+} \mathrm{T}$ cell & {$[88]$} \\
\hline & NY-ESO-1 & SLLMWITQV & $\mathrm{CD}^{+} \mathrm{T}$ cell & {$[89]$} \\
\hline & MAGE-A3 & FLWGPRALV & $\mathrm{CD}^{+} \mathrm{T}$ cell & {$[90]$} \\
\hline & Tyrosinase $_{1-9}$ & MLLAVLYCL & $\mathrm{CD}^{+} \mathrm{T}$ cell & {$[91]$} \\
\hline & Tyrosinase $_{368-376}$ & YMDGTMSQV & $\mathrm{CD}^{+} \mathrm{T}$ cell & {$[91]$} \\
\hline & MART-1 $26-35$ & EAAGIGILTV & $\mathrm{CD}^{+} \mathrm{T}$ cell & {$[92]$} \\
\hline & gp $100_{280-288}$ & YLEPGPVTA & $\mathrm{CD}^{+} \mathrm{T}$ cell & [93] \\
\hline & gp100 $209-217$ & IMDQVPFSV & $\mathrm{CD}^{+} \mathrm{T}$ cell & {$[94]$} \\
\hline & HGP100 & KVPRNQDWL & $\mathrm{CD}^{+} \mathrm{T}$ cell & {$[95]$} \\
\hline & TRP2 & SVYDFFVWL & $\mathrm{CD}^{+} \mathrm{T}$ cell & {$[96]$} \\
\hline & Survivin- $2 \mathrm{~B}_{80-88}$ & AYACNTSTL & $\mathrm{CD}^{+} \mathrm{T}$ cell & {$[97]$} \\
\hline & E75 & KIFGSLAFL & $\mathrm{CD}^{+} \mathrm{T}$ cell & [98] \\
\hline & WT1Pep427 & $\begin{array}{c}\text { RSDELVRHH } \\
\text {-NMHQRNMTKL }\end{array}$ & $\mathrm{CD} 4^{+} \mathrm{T}$ cell & [99] \\
\hline & $\mathrm{E}_{11-20}$ & YMLDLQPETT & $\mathrm{CD}^{+} \mathrm{T}$ cell & {$[100]$} \\
\hline & $\mathrm{E}_{86-93}$ & TLGIVCPI & $\mathrm{CD}^{+} \mathrm{T}$ cell & {$[101]$} \\
\hline & $\mathrm{E}_{43-57}$ & GQAEPDRAHYNIVTF & $\mathrm{CD}^{+}, \mathrm{CD}^{+} \mathrm{T}$ cell & {$[102]$} \\
\hline & $\mathrm{E}_{49-57}$ & RAHYNIVTF & $\mathrm{CD}^{+} \mathrm{T}$ cell & {$[103]$} \\
\hline & $\mathrm{E}_{48-54}$ & PDRAHYNI & $\mathrm{CD}^{+} \mathrm{T}$ cell & {$[104]$} \\
\hline & OFA 2 & ALCNTDSPL & $\mathrm{CD}^{+}, \mathrm{CD}^{+} \mathrm{T}$ cell & {$[105]$} \\
\hline \multirow{5}{*}{ Vaccine adjuvants } & Q11 & QQKFQFQFEQQ & - & {$[106]$} \\
\hline & KFE8 & FKFEFKFE & - & {$[107]$} \\
\hline & Hydrogel & Nap- $G^{D} F^{D} F^{D} Y^{D}$ & - & {$[108]$} \\
\hline & Hydrogel & Nap- $G^{D} F^{D} F^{D} Y^{D} K$ & - & {$[109]$} \\
\hline & Hydrogel & $G^{D} F^{D} F^{D} Y$ & - & {$[110]$} \\
\hline
\end{tabular}

mirror-image phage display technology. Mirror-image phage display technology allows for screening D-clones for binding L-targets by using a chemical synthesized D- peptide bait. Starting from the immunoglobulin-like variable $(\mathrm{Ig}-\mathrm{V})$ domain, the authors designed a $\mathrm{D}$-version of the folded IgV domain ( $\left.{ }^{\mathrm{I}} \mathrm{IgV}{ }^{\mathrm{PD}-\mathrm{Ll}} 9\right)$ serving as the bait 
peptide in mirror-image phage display. Screening a duodecimal peptide library displayed on M13 phage allowed the authors to select two D-sequences $\left({ }^{\mathrm{D} P P A} 1\right.$ : NYSKPTDRQYHF, ${ }^{D}$ PPA 2: KHAHHTHNLRLP) with the highest frequency. Surface plasmon resonance spectroscopy estimated the binding constants $\left(K_{\mathrm{D}}\right)$ of ${ }^{\mathrm{D}}$ PPA 1 and ${ }^{\mathrm{P} P A} 2$ with human PD-1 to be 0.51 and $1.13 \mu \mathrm{mol} \mathrm{L}^{-1}$, respectively. Flow cytometry experiments showed that ${ }^{\mathrm{D}}$ PPA 1 exhibited an advanced capability for inhibiting PD-1/PD-L1 interaction compared to ${ }^{\mathrm{D} P P A} 2$. In vivo experiments revealed the inhibition of the growth of CT26 cells implanted in $36 \mathrm{Balb} / \mathrm{c}$ mice by the ${ }^{\mathrm{D} P P A} 1$ administration potentially due to the activation of the antitumor immune system. Therefore, the anti-hydrolysis D-peptide has the potency as a small molecular drug for cancer immunotherapy, which has been currently utilized in many preclinical studies.

On the basis of the yeast-surface display technology, Maute et al. [82] developed competitive peptide antagonists, i.e., HAC-I (HVIHEGTVVI) and HAC-V (HVVHEGTVVI), with a high affinity with PD-L1 using a two-library strategy. In this approach, the first generation library derived from the domain of human PD-1 at the interacting interface with PD-L1 allowed for identification of mutational residues governing the high affinity, whereas the second generation library determined the optimal combination of the residues. As a consequence, two sequences of HAC peptides that are merely different with an isoleucine or valine residue at position 41 were produced, and the resulting HAC sequences enable to bind with PD-L1 with a $K_{\mathrm{D}}$ value of approximately $100 \mathrm{pmol} \mathrm{L}^{-1}$. Therapeutic studies showed that the HAC peptides possessed the ability to treat both small and large tumors. In particular, radiolabeling the HAC peptides led to positron emission tomography imaging of the presence of PD-L1 in tumors, thus allowing for direct immune diagnostics.

Based on an alternating random and focused library screening strategy of bacterial surface display technology, $\mathrm{Zhu}$ and coworkers [83] discovered a targeting PD-L1 peptide (TPP-1, SGQYASYHCWCWRDPGRSGGSK) with a high associating affinity with PD-L1 and the capability to inhibit the PD-1/PD-L1 interaction. Both in vitro and in vivo assays revealed that treatment of tumors with TPP-1 activates $\mathrm{T}$ cells and elicited immune responses, thus demonstrating the inhibitory potency in cancer immunotherapy. Andersen and coworkers [84] designed and synthesized a $\mathrm{T}$ cell epitope derived from PD-L1, termed as PDLong-1 (FMTYWHLLNAFTVTVPKDL), which contains a PD-L1-derived
$\mathrm{CD}^{+} \mathrm{T}$ cell epitope ( $\mathrm{PDL1}_{15-23}$, LLNAFTVTV). The authors found that co-stimulation of dendritic cell (DC)based vaccination with PDLong-1 led to significant increase of the number of $\mathrm{T}$ cells. This finding demonstrates that reactivation of $\mathrm{PD}-\mathrm{L} 1$ associated $\mathrm{T}$ cells potentially allows for direct modulation of DC vaccination immunogenicity. In addition, starting from the macrocyclic peptide PD-L1 inhibitors developed by Bristol-Myers Squibb, Magiera-Mularz et al. [85] investigated the affinities of three macrocyclic peptides, i.e., peptide-57, peptide-71, and peptide-99, binding with PD-L1. The authors found that all the three macrocyclic peptides inhibited the PD-1/PD-L1 interaction and their affinities with PD-L1 were estimated in an order of peptide-71 > peptide-57 > peptide-99. These limited examples demonstrate that peptides are burgeoning checking point blockades with great potency in modulating the immune system. Combining peptide inhibitors with the peptide nanocarriers leads to a promising strategy towards cancer immunotherapy with low side effect.

\section{PEPTIDE-BASED CANCER VACCINES}

As another representative method of immunotherapy, cancer vaccines target activation of host immune system protecting against cancer cells by using tumor cell-associated antigens. Due to the antigen-specific immune responses and long-term immune memory, cancer vaccines exhibit great potential in cancer immunotherapy. Vaccination probably is the most classical immunotherapy approach and has been utilized broadly through ancient to modern medicine [111]. In conventional cancer vaccines, tumor cell-associated antigens collected from different types of cancer cells are used to stimulate the immune cells like B-cells and T-cells. During vaccination, the injected vaccines can be up-taken by antigen-presenting cells through either endocytosis initiated by binding with Toll-like receptors (TLR) or phagocytosis directly (Fig. 3) [112]. Within the APC cells, the up-taken antigens were degraded into short peptides based on proteasome-mediated processes. Displaying the resulting peptides on the APC cell surface via association with major histocompatibility complex (MHC) class I or II receptors leads to activation of immune responses. In the case of the MHC class I pathway, association of antigens with MHC and T cell receptors (TCR) results in production of $\mathrm{CD}^{+}$cells eliciting cellular immune responses involving cytotoxic T-lymphocyte (CTL) cells [113]. However binding antigens with the MHC class II receptors activates the T-helper cells that further cause production of B cells for humoral immunity or CTL cells 


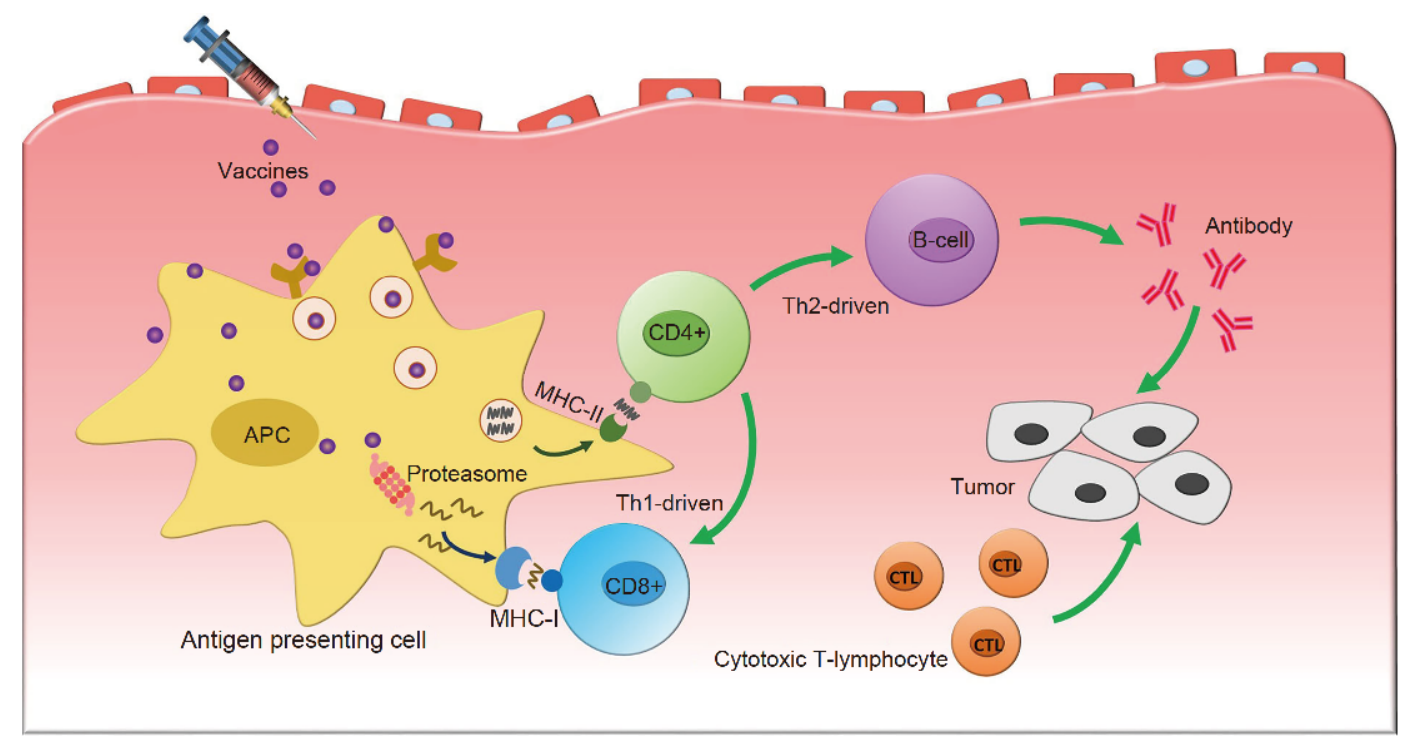

Figure 3 Schematic representation of immune response pathways for vaccines. Vaccines are up-taken by antigen-presenting cells (APCs) through either endocytosis initiated by binding with Toll-like receptors (TLR) or phagocytosis directly. Degradation of vaccines into short peptides allows for displaying on the surface of APCs via association with major histocompatibility complex (MHC) class I or II receptors.

for cellular immunity [114,115]. Traditional cancer vaccines are attenuated organisms or viruses and the subunits of active proteins isolated from viruses. Despite the success in some cancer treatments, traditional vaccination therapy remains challenging primarily arising from contaminant-associated immune risks, poor stability of antigens, difficulties in production and transportation to lymph nodes, and the off-targeting-induced undesired autoimmunity, among others [116].

\section{Peptide antigens}

Recently, short peptides with minimal antigenic epitopes to bind with targeting receptors have been developed as alternative vaccines due to their precise structures and facile production $[117,118]$. Sufficient affinity of antigens for the TLR or MHC receptors is crucial for promoting the production of $\mathrm{CD}^{+}$or $\mathrm{CD}^{+} \mathrm{T}$ cells for immune responses. Due to the limited immunogenicity of short peptide antigens, utilization of peptide adjuvants to assist the activation of the immune response of peptide antigens is a typical strategy and indeed a bunch of peptide adjuvants have been developed thus far. This section briefly summarizes peptide vaccines including design of peptide antigens involving sequences less than 20 amino acids. Due to the long period of peptide vaccine research, the more detailed reviews of peptide vaccines were referred to elsewhere $[111,112]$.

On the basis of the revealed sequences of native pro- teins within organism or virus vaccines, the subunits of these proteins inspire the design of short peptides as potential antigens to induce remarkable and long-term immunity against viruses (Table 1). Dependent on the stimulating pathways, the designed peptide antigens associated with MHC class I receptors towards activation of $\mathrm{CD}^{+} \mathrm{T}$ cells typically consist of $8-10$ amino acids, whereas the antigens loaded by MHC class II receptors and $\mathrm{CD}^{+} \mathrm{T}$ cells usually possess $13-18$ amino acids, though there is no strict limitation on the peptide length [117]. Due to the heterogeneity of the MHC receptors of individual patients, immune tolerance to peptide antigens has been observed in clinical trials. Combining the targeting delivery with the assistance of peptide adjuvants in immune responses renders peptides antigens still promising in cancer immunotherapy [119]. Here we summarize peptide antigens mostly developed within the past decade with an emphasis on the originality of peptides and the underlying immunogenicity mechanism.

Peptide vaccines have been broadly utilized in melanoma immunotherapy [120]. Most melanoma peptide antigens are derived from melanocyte differentiation proteins such as tyrosinase, MART-1 (Melan-A), and glycoprotein 100 (gp100) [121], and predominately promote the production of CTLs for immunity. For instance, tyrosinase, which is the rate-limiting enzyme in melanin synthesis, contains two immunogenic peptides, i.e., tyrosinase $_{1-9}$ and tyrosinase ${ }_{368-376}$ [91]. The tyrosinase $368-376$ 
domain with the replacement of asparagine residue at position 3 with aspartic acid was proved to exhibit the extraordinary immunogenicity. The original MART- $1_{26-35}$ domain [92,122] was employed as antigens in clinical trials to treat melanoma patients as well. In addition, the domain of gp $100_{280-288}$ within protein gp100 [93] that is expressed by both melanoma and healthy melanocytes caused immunogenicity for a large proportion of patients, despite a low amount of CTL production. Combining a vaccine-restricted domain gp100 $209-217$ with an immune activator interleukin-2 (IL-2) improved the immune response against cancer cells. A hydrophilic epitope HGP100 $25-33$ and a hydrophobic epitope within tyrosinerelated protein $2\left(\mathrm{TRP} 2_{180-188}\right)$ are two alternative melanoma-derived antigens. Guo et al. [95] created a nanovaccine formulation by integrating nanoparticles composed of poly(D,L-lactide-co-glycolide) functionalized with antigen HGP100 and adjuvant monophosphoryl lipid with liposomes coated with mannose. Mirkin and coworkers [123] attached antigen HGP100 peptide to immune-stimulatory spherical nucleic acid for vaccine development. Wakabayashi et al. [96] utilized a solid-inoil nanodispersion as nanocarriers for the co-delivery of antigen TRP-2 peptide modified with three lysine residues (KKKGSVYDFFVWL) and adjuvant Resiquimod (R-848). This system exhibited the great capability in inhibiting melanoma growth and suppressing lung metastasis in tumor-bearing mice. Antigens HGP100 and TRP2 were also simultaneously co-encapsulated into hollow mesoporous silica nanoparticles, which was efficient in stimulating dendritic cells (DC) and their maturation and further secreting tumor necrosis factor- $\alpha$ (TNF- $\alpha$ ), IFN- $\gamma$, IL-12 and IL-4 for promoting immunity [124].

Epitopes derived from ovalbumin have been widely used as peptide antigens including $\mathrm{OVA}_{257-264}$, $\mathrm{OVA}_{253-266}$, $\mathrm{OVA}_{250-264}$, and $\mathrm{OVA}_{323-339}$, and conventionally activate $\mathrm{CD} 8^{+}$cytotoxic $\mathrm{T}$ cell immune responses. Utilization of the nanocapsules composed of 60 nonviral E2 subunits of pyruvate dehydrogenase, Wang and coworkers [86] developed a viral-mimicking vaccine scaffold encapsulating antigen $\mathrm{OVA}_{257-264}$ and oligonucleotide adjuvant cytosine-guanine motif $(\mathrm{CpG})$. This multifunctional vaccine platform showed synergistically spatiotemporal delivery of therapeutic agents to DCs and thus enhancing $\mathrm{CD}^{+} \mathrm{T}$ cell production and immune activation. In addition, elongating $\mathrm{OVA}_{257-264}$ epitope to CCYSIINFEKL with two thiol groups allowed for in situ preparation of fluorescent antigen-gold nanoclusters (peptide-AuNCs), which displayed enhanced immune- stimulatory capability [125]. The immunity was further improved by co-loading $\mathrm{CpG}$ adjuvant on the AUNC surfaces. Furthermore, Zhang and coworkers [88] created ultra-small biocompatible nanovaccines functionalized with scavenger receptor class B1 targeting mature DCs, which efficiently delivered peptide antigens including $\mathrm{OVA}_{257-264}, \mathrm{OVA}_{323-339}$, and $\mathrm{HGP100}_{25-33}$ to lymph nodes.

Membrane-binding glycoprotein mucin 1 (MUC1) that plays a critical role in protection of epithelial surfaces and signaling transduction is typically overexpressed with glycosylation mutation in many cancers such as breast and pancreas cancers or myelomas and lymphomas, thus rendering MUC1 immunogenic [126]. This phenomenon inspires the design of peptide antigens capable of inducing MUC1-associated cytotoxic T lymphocyte responses. MUC1 is a type I transmembrane glycoprotein featured with an extracellular domain consisting of a variable number of 20-amino acid repeat sequences (PDTRPAPGSTAPPAHGVTSA) and a high glycosylation level on serine and threonine residues within each tandem repeat. Hence, MUC1-related peptide antigens can be designed based on glycosylation of MUC1 epitopes. For example, Huang et al. [127] designed and synthesized several vaccine candidates via conjugating HGVTSAPDTRPAPGSTAPPA sequence with glycosylated threonine residues at position 9 or 16 to an assembling domain Q11. These B cell epitope-containing vaccines elicited significant cytotoxic $\mathrm{T}$ cell immune responses activated by type I T-helper cells. In addition, Zhao and coworkers [128] also designed MUC1-related antitumor vaccines based on covalently connecting antigen candidate glycopeptide tandem repeat TSAPDTRPAP with an assembling sequence Nap- $G^{\mathrm{D}} \mathrm{F}^{\mathrm{D}} \mathrm{F}^{\mathrm{D}} \mathrm{Y}^{\mathrm{D}} \mathrm{K}$.

In addition to the broadly used melanocyte mutated proteins, ovalbumin, and MUC1, some other immunogenic proteins have also been employed to design peptide vaccines. For instance, an epitope E75 derived from HER2/neu, which is a proto-oncogene expressed in many epithelial cancers, was designed and utilized in breast cancer treatments [98]. A WT1 Pep427 originated from Wilm's tumor protein (WT1) was also discovered as immunogenic antigens and was covalently conjugated with single-wall carbon nanotubes to induce rapid specific IgG responses [99]. New York esophageal squamous cell carcinoma-1 (NY-ESO-1) [89] is an immunogenic cancer testis antigen highly expressed in many human cancers (melanoma, breast cancer) and capable of inducing $\mathrm{T}$ cell-associated immunity. Gazzinelli and coworkers [129] connected antigen NY-ESO-1 and adjuvant CpG DNA to carbon nanotubes (CNT) and developed a 
new anti-cancer vaccine platform. Wang and coworkers [90] employed E2 viral-like capsules to simultaneously encapsulate antigens NY-ESO-1 and HLA-A2 to overcome the low immunogenicity of individual antigens. In addition, the epitopes derived from human papillomavirus $(\mathrm{HPV})$ including HPV16 E7 ${ }_{11-20}[100,101], \mathrm{E} 7_{86-93}$ [101], E7 $7_{43-57}[102,103,130], \mathrm{E}_{49-57}[101,103,131]$, and $\mathrm{E}_{48-54}[103,104]$, or from oncofetal antigen including OFA 1, OFA 2, and OFA 3 [105], have been utilized as antigens for cancer immunotherapy. Extending from these examples, new-generation peptide antigens such as multivalent or multifunctional peptide antigens, peptide cocktail antigens, hybrid peptide antigens, as well as personalized peptide antigens (neoantigens) have attracted broad attention in clinical trials and show great potency for cancer therapy.

\section{Peptide vaccine adjuvants}

Design of vaccine antigens from short peptides benefits from their defined structures and selective targets within the immune system. However, immunogenicity caused by peptide antigens is still insufficient and requires the presence of vaccine adjuvants. On the basis of the deep understanding of anti-tumor immune responses, vaccine adjuvants have been widely used to augment immune responses during vaccination. Despite utilization of many adjuvants like aluminium salts for peptide im- munotherapy thus far, currently available adjuvants including oil emulsions, virosomes, and TLR ligands still suffer from their structural heterogeneity and resulting difficulties in mechanism understanding and administration $[132,133]$. Recently, peptide assemblies have been developed as homogenous peptide antigens predominately due to the capability in displaying multivalent antigens on the surface of peptide assemblies.

The pioneer work of peptide vaccine adjuvants was reported by the Collier group [106], in which the authors attached antigen $\mathrm{OVA}_{323-339}$ to a domain Q11 (QQKFQFQFEQQ) able to undergo self-assembly into well-defined nanofibrils, leading to peptide OVA-Q11 that assembled into well-defined nanofibers under mild condition (Fig. 4). Treating C57BL/6 mice with peptide OVA-Q11 resulted in significant increase of the population of IgG titers in serum, thus enhancing the immunogenicity. This enhanced immunogenicity of OVAQ11 was potentially attributed to the multivalent surface display of the epitope on the fibrils. More detailed studies revealed the lack of cytokine responses and an elevated IgM response induced by OVA-Q11, indicative of an immunogenic mechanism independent on $\mathrm{T}$ cell response. The Q11 domain was also used to connect MUC1-derived epitopes with varied glycosylated threonine residues to develop self-adjuvanting antigens from nanofibrils with B cell epitopes displayed on their surface. a

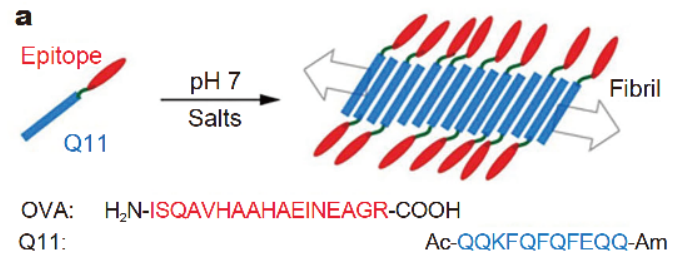
O-Q11: $\mathrm{H}_{2} \mathrm{~N}$-ISQAVHAAHAEINEAGR-SGSG-QQKFQFQFEQQ-Am

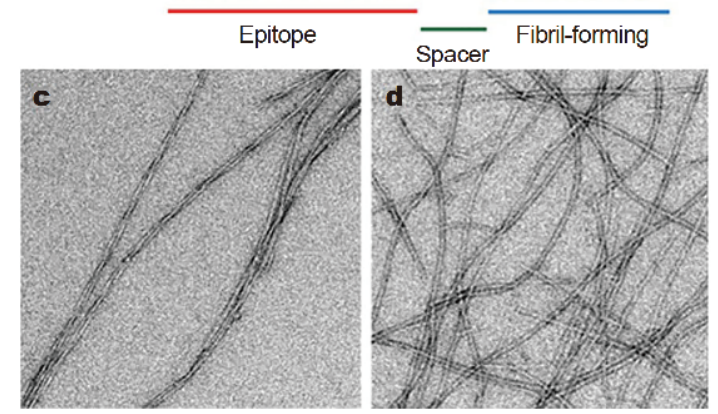

b

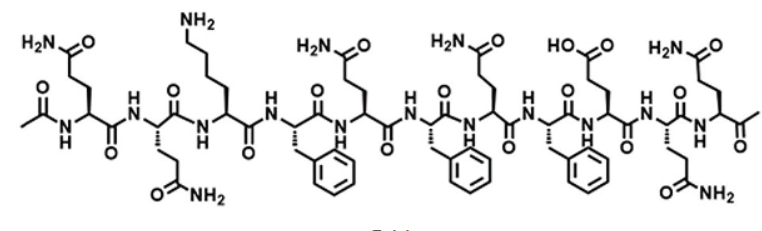

Q11
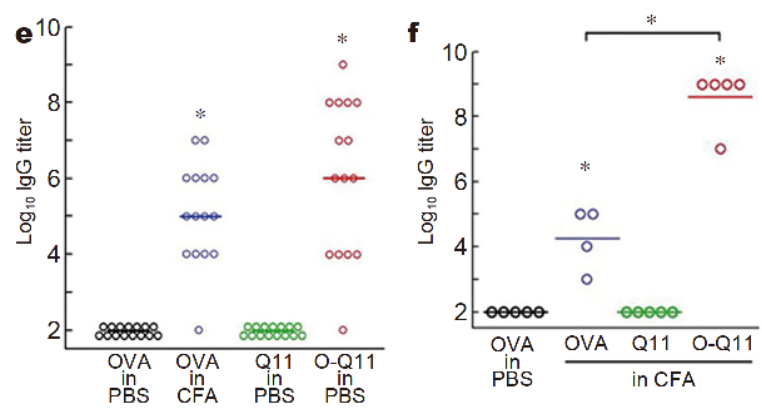

Figure 4 Peptide assemblies as vaccine adjuvants. (a) Schematic illustration of formation of antigen-displaying peptide nanofibrils and the design of sequences consisting of antigen $\mathrm{OVA}_{323-339}$ and assembling domain Q11. (b) Chemical structures of Q11. TEM images of nanofibrils formed by peptide Q11 (c) and O-Q11 (d). (e) Expression of IgG induced by fibrillized Q11 domains compared to traditional complete Freund's adjuvant (CFA). (f) Improved secretion of IgG titers induced by OVA, Q11, and O-Q11 in the presence of CFA. ${ }^{*} p<0.01$. Reproduced with permission from Ref. [106]. Copyright 2010, National Academy of Sciences. 
Alternative to Q11 domain, Collier and coworkers [107] also connected antigen $\mathrm{OVA}_{323-339}$ to a assembling sequence KFE8 (FKFEFKFE), leading to OVA-KFE8 that formed nanofibrils and activated strong antibody responses analogue to OVA-Q11. Furthermore, Rudra and coworkers [134] changed the natural $\mathrm{D}$-amino acids to $\mathrm{L}$ amino acids in Q11 domain and investigated the enantiomeric effect of nanofibrils on immune response of OVA epitopes. On the basis of characterization of enantiomeric nanofibrils, the authors discovered that compared to the L-counterparts, nanofibrils composed of Damino acid sequences enhanced antibody responses and prolonged antigen-presentation in mice, suggestive of the advanced performance of $\mathrm{D}$-peptides in vaccination and also the stereochemistry-associated biomaterials in modulation of the immune system. These results demonstrate that utilization of peptide assemblies as homogenous peptide vaccine adjuvant allows for facilitation of vaccination.

In addition to peptide assemblies, Yang and coworkers [135] created hydrogels composed of peptide assemblies to develop vaccine adjuvants, which might simplify vaccine administration and improve the biosafety of adjuvants (Fig. 5). The authors investigated the enantiomeric effect of the resulting hydrogels on vaccination by synthesizing the peptide gelators consisting of either D- or L-amino acids, i.e., Nap-GFFpY-OMe and Nap- $\mathrm{G}^{\mathrm{D}} \mathrm{F}^{\mathrm{D}} \mathrm{F}^{\mathrm{D}} \mathrm{pY}-\mathrm{OMe}$, which underwent hydrogelation promoted by alkaline phosphatase (ALP)-induced dephosphorylation. Co-assembling of the gelators with OVA protein maintained the hydrogelating behavior, implying the efficient up-taking of OVA within the hydrogels. In vivo studies revealed that both the two enantiomeric hydrogel adjuvants effectively caused production of immune antibody and secretion of cytokines, due to the enhanced cellular uptake of antigens, accumulation of antigen at lymph nodes, maturation of dendritic cells, and formation of germinal centers. In particular, the authors found that the D-peptide hydrogels possessed a better performance in accumulating OVA and preventing tumor growth, compared to the Lcounterpart hydrogels.

To further avoid the difficulty in preparing vaccine adjuvants caused by enzymatic hydrolysis, the authors developed hydrogel adjuvants directly from enantiomeric peptide gelators Nap-GFFY and Nap- $G^{\mathrm{D}} \mathrm{F}^{\mathrm{D}} \mathrm{F}^{\mathrm{D}} \mathrm{Y}$ [108]. The thixotropic feature of the resulting hydrogels allowed for efficient encapsulation of antigen OVA. In addition, the hydrogels also encapsulated X-ray attenuated tumor cells serving as antigen therapeutics to suppress tumor growth and prolong the survival of tumor-bearing mice through the $\mathrm{CD} 8^{+} \mathrm{T}$ cell activation pathway. Analogue to the enzyme-instructed hydrogelation, Nap- $G^{\mathrm{D}} \mathrm{F}^{\mathrm{D}} \mathrm{F}^{\mathrm{D}} \mathrm{Y}$ hydrogels exhibited advanced capability in activation of immune response compared to the L-peptide hydrogels. On the basis of the concept of hydrogel adjuvants, a series
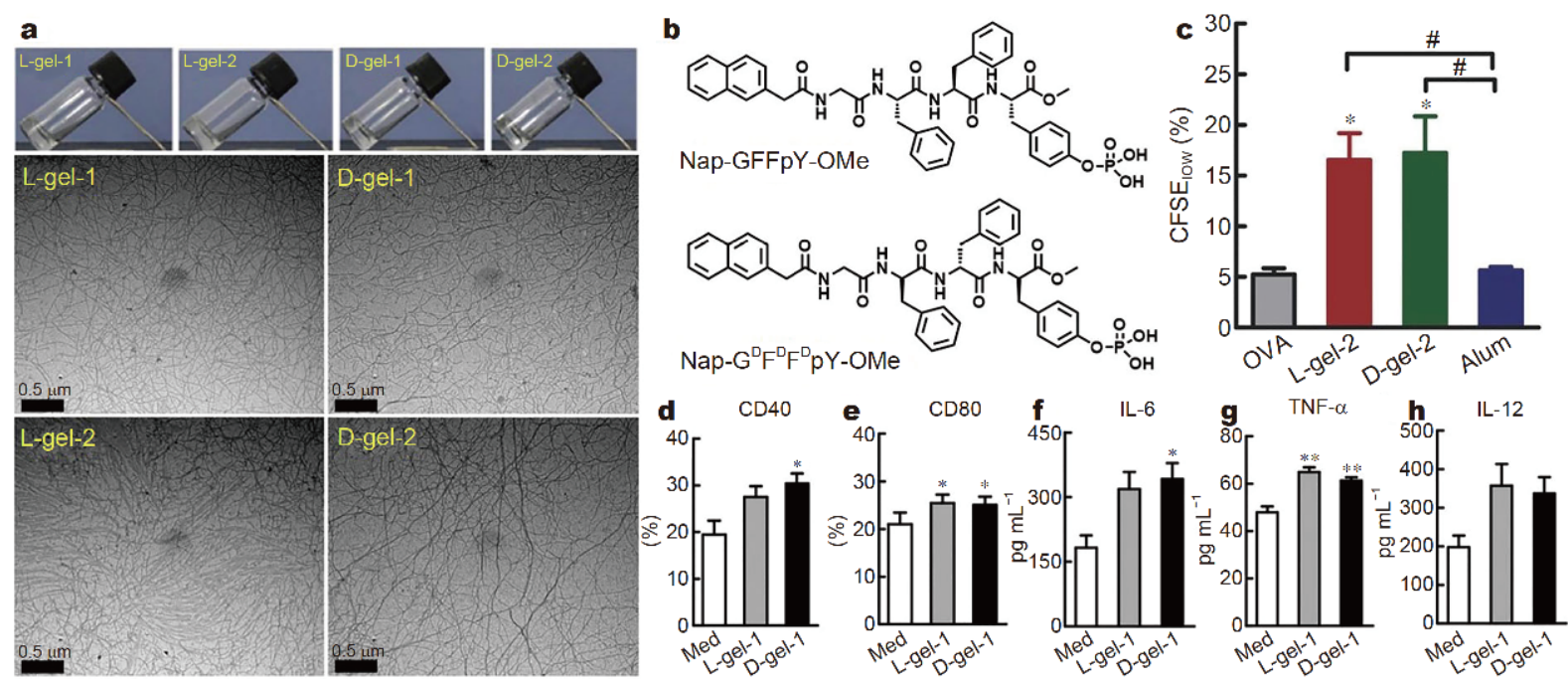

Figure 5 Peptide hydrogels as vaccine adjuvants. (a) Optical and TEM images of hydrogels prepared by phosphatase-induced hydrolysis of NapGFFpY-OMe and Nap- $\mathrm{G}^{\mathrm{D}} \mathrm{F}^{\mathrm{D}} \mathrm{F}^{\mathrm{D}} \mathrm{pY}$-OMe in the absence ( L-gel-1, D-gel-1) or presence (L -gel-2, D-gel-2) of antigen OVA. (b) Chemical structures of Nap-GFFpY-OMe and Nap-G ${ }^{\mathrm{D}} \mathrm{F}^{\mathrm{D}} \mathrm{F}^{\mathrm{D}} \mathrm{pY}$-OMe. (c) The numbers of germinal centers and B-cell follicles induced by different vaccines. (d-h) Production of CD40 (d) and CD86 (e) based on maturation of bone marrow dendritic cells and expression of cytokine IL-6 (f), TNF- $\alpha$ (g), and IL-12 (h) treated with medium (Med) or L-/D-gel vaccines. ${ }^{*} p<0.05$. Reproduced with permission from Ref. [135]. Copyright 2016, John Wiley and Sons. 
of peptide gelators derived from the Nap-GFFY or Nap$G^{D} F^{D} F^{D} Y^{D}$ sequences have been developed for vaccine adjuvants. For example, tailing Nap- $G^{D} F^{D} F^{D} Y^{D}$ with either a positively or negatively charged residue at C-terminus led to Nap- $G^{\mathrm{D}} \mathrm{F}^{\mathrm{D}} \mathrm{F}^{\mathrm{D}} \mathrm{Y}^{\mathrm{D}} \mathrm{K}$ and Nap- $\mathrm{G}^{\mathrm{D}} \mathrm{F}^{\mathrm{D}} \mathrm{F}^{\mathrm{D}} \mathrm{Y}^{\mathrm{D}} \mathrm{E}$ [109]. When encapsulating OVA protein, the hydrogels composed of the positively charged peptides displayed the better capability in inducing immune responses compared to the negatively charged one, potentially attributed to the efficient encapsulation of OVA. In another example, the authors replaced naphthalene unit with nonsteroidal anti-inflammatory drugs at the $\mathrm{N}$-terminus of $G^{D} F^{D} F^{D} Y$, resulting in several drug-modified vaccine adjuvants [110]. Combining the anti-inflammatory property of drugs, the hydrogels up-taking OVA showed the extraordinary capability in elimination of tumor in mice. Alternative to OVA protein, incorporation of MUC1 epitopes into Nap- $G^{D} F^{D} F^{D} Y^{D} K$ sequence allows for enhancement of the immunogenicity of antigen MUC1 [128].

\section{PEPTIDE ASSMEMBLIES IN CANCER IMMUNOTHERAPY}

Despite their success in immune activation in some preclinical studies and clinical trials, peptide immune therapeutics still suffer from the low immunogenicity in clinical trials. In principle, shortening the sequences of native proteins to partial epitopes significantly weakens the affinity of peptides to corresponding targeting receptors, and also increases the possibility of the enzymatic degradation of short peptides, which further lowers the circulation life-time and accumulation of peptides around lymph nodes. In addition to these drawbacks, the heterogeneity of APC cells of patients further leads to the challenge in effective immunogenicity of peptide therapeutics. Based on these considerations, exploration of additional functions of peptides in cancer immunotherapy becomes essential.

Self-assembly of peptides into well-defined nanostructures with morphologies ranging from nanoparticles [136], nanofibers [137], nanoribbons [138], nanotubes [139], to hierarchical networks, driven by noncovalent interactions including hydrophobic interactions, hydrogen bonding interactions, and $\pi-\pi$ stacking interactions, has great potency to address these issues. Basically, the resulting nanostructures are ideal platforms to deliver and display peptide therapeutics due to their unique structural properties such as biocompatibility and biodegradability $[132,140]$. Incorporation of peptide therapeutics into peptide nanostructures allows for prolonging the circu- lation of therapeutics and increasing the affinity with targeting receptors arising from multivalent effect. In addition, the passive or active targeting capability of peptide nanostructures facilitates the accumulation of therapeutics at tumor sites. These advantages of peptide assemblies give rise to the great potential of peptide-based biomaterials as vaccine adjuvants for enhancement of antigen immune responses or the platforms for delivery of immunotherapeutic agents. This section covers cancer immunotherapy using peptide assemblies in cancer vaccination including vaccine adjuvants or delivery systems of immune therapeutics including genes, vaccines, antigens, checkpoint blockades, or their combinatorial drugs with conventional cancer therapeutics.

\section{Peptide assemblies in vaccination delivery}

Despite the great potential of cancer vaccines in humoral and cellular immune responses, many clinical trials of vaccination still suffer from the low immunogenicity. This potentially results from several reasons, such as immunosuppression, poor $\mathrm{T}$ cell infiltration, and low production of $\mathrm{T}$ cell. Development of novel delivery scaffolds able to target antigen-presenting cells and facilitate antigen production allows for improvement of the immunogenicity, thus potentially leading to positive clinical performance. Compared to conventional biomaterials, the precisely customizable properties of peptide assemblies render their great potential for serving as delivery systems for cancer vaccines. Extending from the conventional functions of peptide assemblies as delivery platforms, modulating the aggregating features of peptide therapeutics also allows for establishment of self-delivering systems, termed as drug amphiphiles [141]. In addition to the succeeded advantages of peptide delivery systems, assemblies of drug amphiphiles increase the density of antigens on the surface of platforms and also eliminate the content of useless components in drug formulation, thus lowering the biosafety, decreasing production cost, and simplifying drug administration. Combining the traditional strategy with the burgeoning approaches, it is promising to develop peptide delivery system for cancer vaccines to improve their immunogenicity.

In the case of drug amphiphiles, Tirrell and coworkers [87] developed the pioneering work of antigen amphiphiles by attaching two palmitic chains to a cytotoxic Tcell epitope from ovalbumin, i.e., $\mathrm{OVA}_{253-266}$ (SIINFEKL), leading to antigen amphiphile DiC16-OVA (Fig. 6). Selfassembly of DiC16-OVA amphiphiles led to formation of cylindrical micelles with a diameter of approximately 
$8 \mathrm{~nm}$ and a length distribution mainly ranging from 200 to $500 \mathrm{~nm}$. The resulting cylindrical micelles consisted of a hydrophobic core composed of alkyl tails and a hydrophilic surface displaying antigen epitopes. Under physiological condition, the antigen micelles are stable with a lifetime over hours, which is beneficial for transportation and accumulation of antigen to lymph nodes. Cellular assays showed that incubation of cells in the presence of DiC16-OVA assemblies did not lead to stimulation of DC cells for immune response. In vivo studies showed that treatment of tumor-bearing mice induced suppression of tumor growth and prolonging the survival of mice through activation of cytotoxic T-cell immune response. This concept demonstrated that selfassembly of antigen amphiphiles into nanostructures is an efficient strategy for antigen delivery and development of self-adjuvanting antigen systems.

In addition to delivering single cancer vaccines, peptide assemblies were utilized to transport multiple antigens with tunable dose ratios. For this purpose, Collier and coworkers [142] developed a strategy for creation of peptide nanofibrils able to integrate multiple proteins while maintaining their independent conformation and bioactivity (Fig. 7). The proteins were fused with a tag sequence MALKVELEKLKSELVVLHSELHKLKSEL, termed as $\beta$ Tail that undergoes a slow conformational transition from an $\alpha$-helix to a $\beta$-sheet in solution. Simultaneously dissolving the $\beta$ Tail fusion protein, i.e., $\beta$ Tail-GFP, with peptide Q11 that rapidly assemblies into nanofibers led to efficient integration of fusion proteins into nanofibers. The dose of the integrated proteins within nanofibers was precisely tuned based on the concentration of proteins in solution. This method was applied to integrate different proteins, i.e., $\beta$ Tail-GFP and $\beta$ Tail-cutinase, into nanofibers with a controllable molar ration, in which individual protein-associated antibody titers were induced. Combining the adjuvanticity of single fusion protein with the capability for precisely integrating multiple proteins, a tailorable multi-antigen vaccine scaffold with controllable antigenic dominance based on
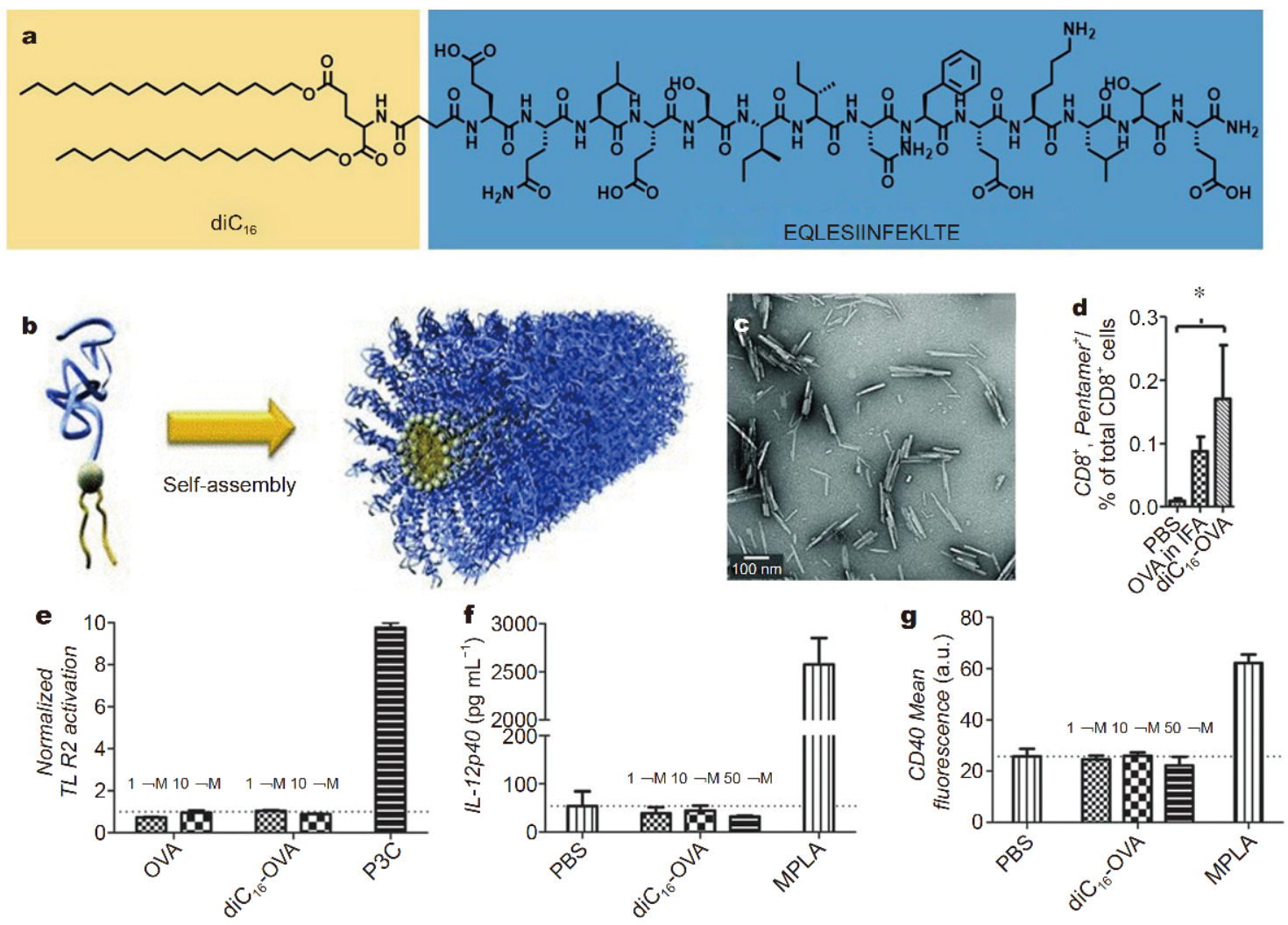

Figure 6 Self-assembled antigen amphiphiles. (a) Chemical structure of peptide amphiphile $\mathrm{diC}_{16}$-OVA composed of OVA $\mathrm{A}_{253-266}$ and two palmitic tails. Schematic representation of self-assembly of $\mathrm{diC}_{16}$-OVA into cylindrical micelles (b) and their TEM image (c). (d) Production of CD8 ${ }^{+}$cells induced by different treatments. ${ }^{*} p<0.05$. Expression of cytokine TLR2 in transfected HEK cells (e), IL-12p40 in DC cells (f), and CD40 in DC cells (g) induced by various treatments. Reproduced with permission from Ref. [87]. Copyright 2012, John Wiley and Sons. 

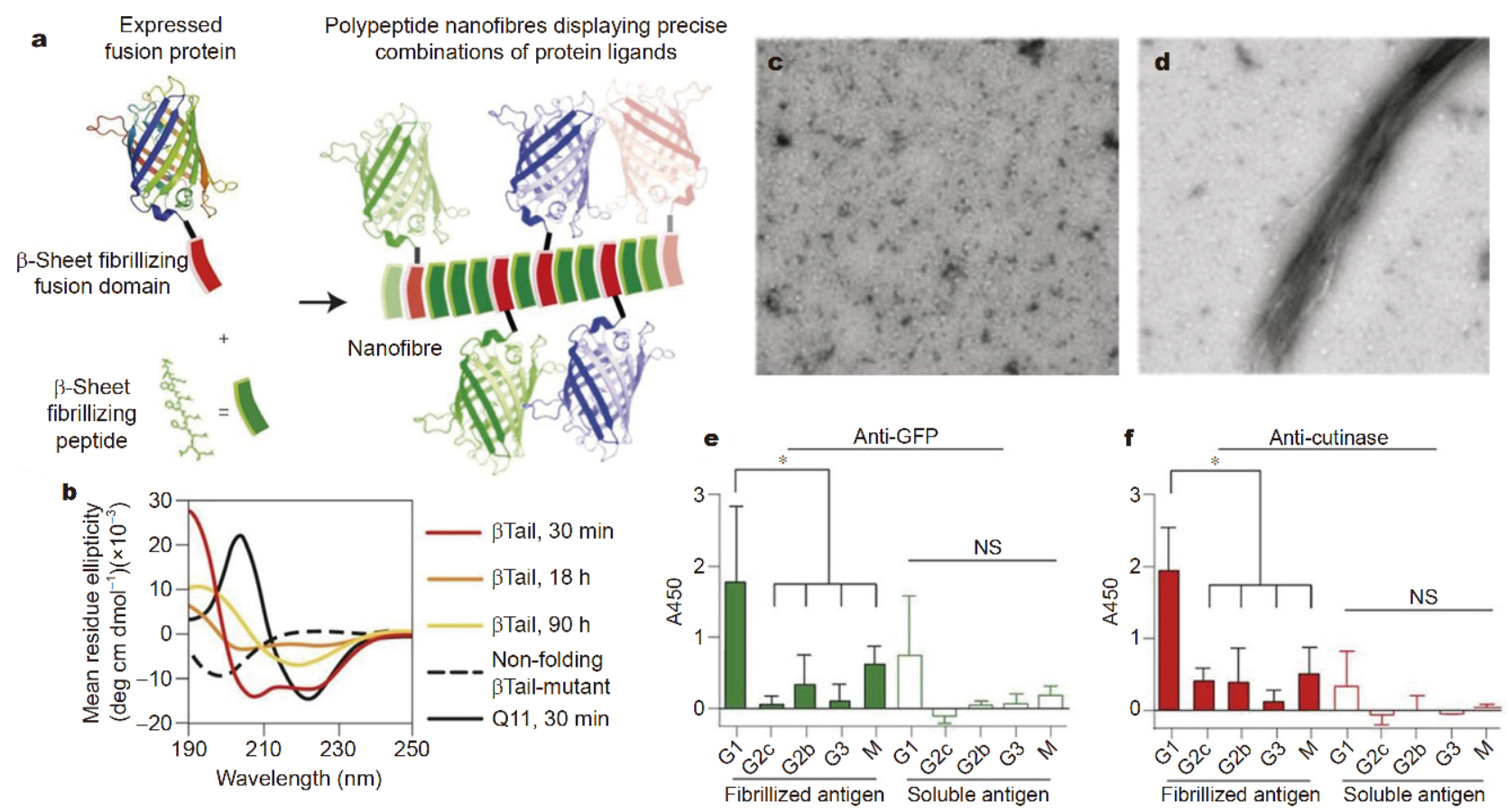

Figure 7 Supramolecular nanomaterials with integrated multiple proteins. (a) Schematic illustration of integration of multiple engineered fusion proteins with a $\beta$ Tail domain into Q11 nanofibers. (b) CD spectra of peptide $\beta$ Tail at different aging times, indicative of the conformational transition from $\alpha$-helix to $\beta$-sheet, as well as CD spectra of Q11 and $\beta$ Tail-mutant. TEM images of self-assemblies of peptide $\beta$ Tail before (c) and after (d) the conformational transition. (e) Antibody polarization towards IgG1 in mice immunized with proteins GFP and cutinase co-fibrillized nanofibers, which is consistent with that immunized with individual protein. Reproduced with permission from Ref. [142]. Copyright 2014, Nature Publishing Group.

the dose of each antigen and the booster formulation was established. The multi-antigen vaccine platforms exhibit advantages of simultaneous immunity against different pathogens and high affinity for single pathogen, thus leading to great potential in cancer vaccination.

\section{Peptide assemblies in combinatorial immunotherapy}

Due to the complicated underlying mechanism for host immune operation as well as the phenotypic heterogeneity of individuals, modulating immune responses by single immune therapeutic approach is insufficient in most cases, thus leading to low immunogenicity and limiting the applications of cancer immunotherapy in clinical trials. Simultaneously integrating different therapeutics in immunotherapy has been considered to be efficient for enhancing immune responses through various pathways [143-145]. In addition, the relative long period for immune responses compared with the instant treating effect in conventional therapies such as chemotherapy and phototherapy and the usual cycle involving repetitive formulation administration for immune responses further limit the clinical application of cancer immunotherapy to the patients under the late stage of cancers. Combining conventional therapy of tumors with immunotherapy enhances the immune responses and has synergistic effects for curative metastatic cancer treatment $[146,147]$. Therefore, to efficiently prolong the survival of patients, combinatorial treatments involving both conventional therapies and immunotherapy have been also developed. Due to their intrinsic capability for both covalently and non-covalently up-taking cargoes, peptide assemblies have been broadly utilized in combinatorial immunotherapies. This section covers the progress in utilization of peptide assemblies as platforms for co-delivery of multiple therapeutics towards combinatorial immunotherapy achieved recently. It is worth noting that the approaches of co-administrated immunotherapy or using administration booster [38], rather than the codelivery strategy, will not be discussed here. In addition, the strategies using polypeptides as delivery vehicles [50] will also not be covered due to the focus on the selfassembly of peptides.

\section{Combinatorial immunotherapy}

Peptide nanostructures or hydrogels composed of peptide assemblies enable to encapsulate many immune ther- 
apeutics ranging from large cargoes like immunogenic cells and antibodies, to small objects like peptides and small synthetic drugs. In particular, the hydrogelating behavior of peptide assemblies simplifies the preparation of injectable formulation and increases the maintenance of therapeutics at tumor sites during administration. In addition, peptide assemblies could be manipulated under the stimuli associated with cancer biomarkers or tumor microenvironment, thus potentially allowing for spatial and temporal on-demand release of therapeutics. Hence, utilization of peptide assemblies to co-deliver multiple immune therapeutics exhibits the great potential for enhancing immune responses in cancer immunotherapy. Here we summarize several most recent examples using peptide assemblies to co-deliver therapeutics for multiple immunotherapy.

Based on the co-delivering strategies, peptide assemblies were used to co-deliver short peptide antigens and small drug inhibitors. Nie and coworkers [148] created a peptide assembling nanoparticles based on co-assembly of an amphiphilic peptide containing with a 3-diethylaminopropyl isothiocyanate (DEAP) segment, a domain PLGLAG cleavable by matrix metalloproteinase-2 (MMP2 ), and a short D-peptide antagonist ( $\left.{ }^{\mathrm{D} P P A}-1\right)$, with a drug NLG919 as the inhibitor for idoleamine 2,3-dioxygenase (IDO) that is an immunosuppressive enzyme due to its capability to hydrolyze L-tryptophan to L-kynurenine (Fig. 8). The mild acidic microenvironment induced the structural swelling of the resulting nanoparticles termed as NLG919@DEAP- ${ }^{\mathrm{D} P P A-1}$ due to decrease of the hydrophobicity of DEAP moieties arising from their protonation, thus facilitating the MMP-2 cleavage of PLGLAG domain and collapse of the nanoparticles and thereby release of up-loaded cargoes. Overexpression of MMP-2 by tumor cells allows for spatial release of antagonist ${ }^{\mathrm{D} P P A}-1$ and drug NLG919, which target PD-L1 and IDO, respectively, around tumor sites. Flow cytometric analysis revealed that the cleaved LAG ${ }^{\mathrm{D}}$ PPA-1 domain exhibited strong associating affinity with PD-L1 despite the addition of the three N-terminal residues. Under the mild acidic condition and in the presence of MMP-2, inhibition of IDO expression induced by treatment of NLG919@DEAP- ${ }^{\mathrm{P} P A-1}$ is comparable to free NLG919. Treating melanoma-bearing mice with NLG919@DEAP- ${ }^{\mathrm{D}} \mathrm{PPA}-1$ increased the level of tumor-infiltrated CTL and thereby efficiently inhibiting tumor growth.

Extending from small therapeutic molecules, peptide assemblies have been employed to co-deliver immune cell vaccines with other therapeutics. In this context, $\mathrm{Li}$ and coworkers [149] developed a personalized cancer vaccine (PVAX) via simultaneously encapsulating attenuated tu-

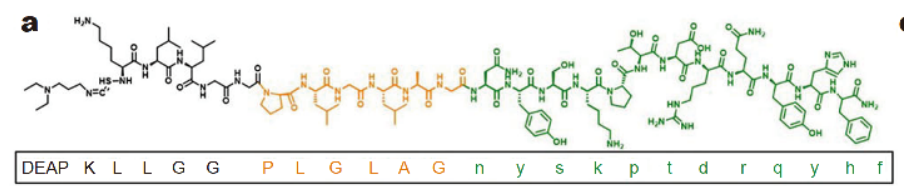

b

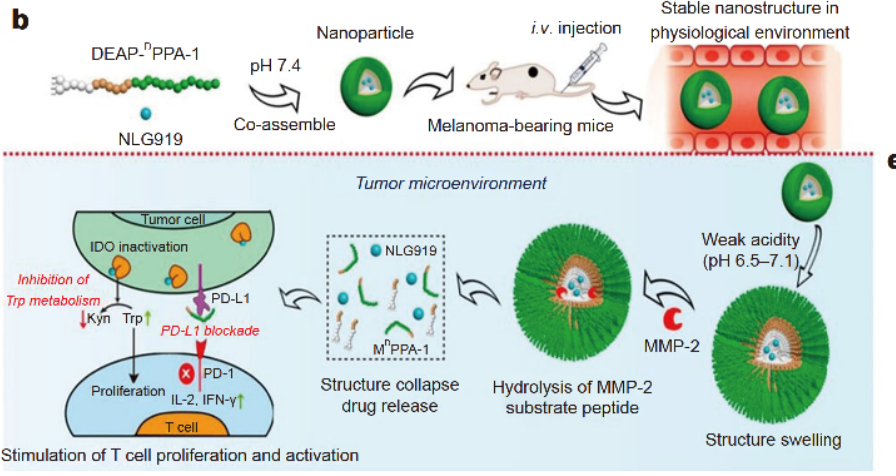

c

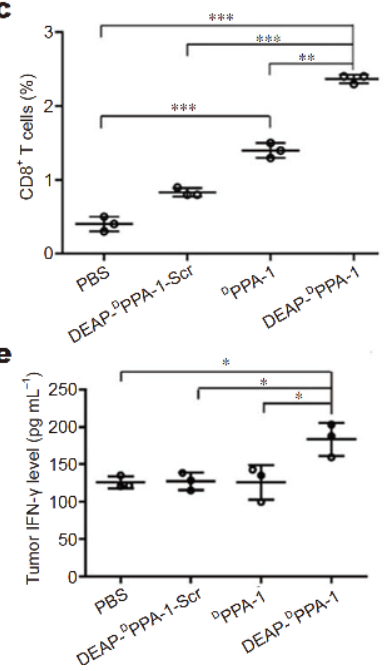

d

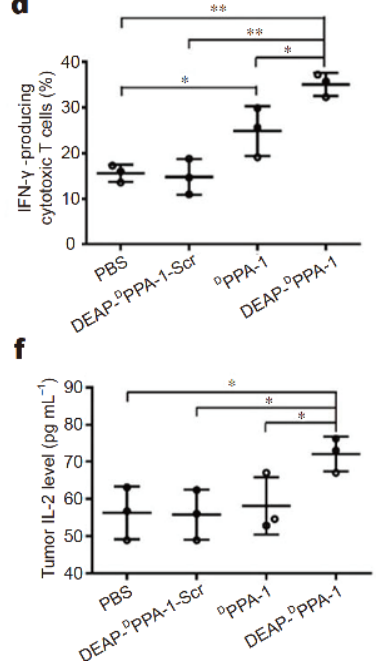

Figure 8 Sequentially responsive peptide assemblies for combinatorial anti-PD-L1 and anti-IDO immunotherapy. (a) Chemical structure of DEAP${ }^{D}$ PPA-1 consisting of a MMP-2-cleavable fragment PLGLAG as a linker to form the hydrophobic domain and a D-peptide antagonist ${ }^{\mathrm{D}}$ PPA-1. (b) Schematic illustration therapeutic mechanism of NLG919@DEAP- ${ }^{\mathrm{D} P P A}-1$ nanoparticles created from assembly of DEAP- ${ }^{\mathrm{D}} \mathrm{PPA}-1$ and encapsulation of IDO inhibitor NLG919. Production of CD8 ${ }^{+} \mathrm{T}$ cells (c) and IFN- $\gamma$-producing cytotoxic T cells (d) induced by immunization of NLG919@DEAP${ }^{\mathrm{D}}$ PPA-1 nanoparticles in tumors after treated on day 12. Expression of cytokines IFN- $\gamma$ (e) and IL-2 (f) in mice estimated by ELISA in extracts of isolated tumors 12 days after treatment termination. ${ }^{*} p<0.05,{ }^{* *} p<0.01,{ }^{* *} p<0.001$. Reproduced with permission from Ref. [148]. Copyright 2018, American Chemical Society. 
mor cells and checkpoint blockades within peptide hydrogels, leading to FK@IQ-4T1 vaccine (Fig. 9). The attenuated tumor cell was collected from mouse 4T1 breast tumor xenografts and cultured in a Foxp2 fixation and permeabilization buffer prior to hydrogel encapsulation. A small drug, i.e., JQ 1, was employed as the inhibitor for bromodomain and extraterminal protein BRD4, which caused immune tolerance by controlling intratumoral expression of PD-L1. The peptide hydrogels were prepared from one sequence Fmoc-KCRGDK (FK) containing with two Fmoc groups on the N-terminal lysine residue to induce self-assembly of peptides and stabilize the hydrogels involving $\pi, \pi$-stacking interactions, whereas the RGD segment facilitates tumor-targeting delivery of therapeutics. To promote release of therapeutics, a fluorescent dye ICG exhibiting high photothermal conversion efficiency was co-loaded to promote the release of 4T1 and JQ1 based on the morphological transition of peptide assemblies induced by the hyperthermia effect upon exposure to laser irradiation. Combined flow cytometric and enzyme-linked immunosorbent assays revealed that FK@IQ-4T1 vaccines promoted in vivo and in vitro DC maturation, elicited $\mathrm{CD}^{+} \mathrm{CTL}$ immune responses and blocked the PD-1/PD$\mathrm{L} 1$ association via suppressing BRD4 activation. In vivo experiments further demonstrated the capability of PVAX vaccine in prevention of postsurgical tumor recurrence and metastasis via eliciting memory immune responses, indicative of a robust cancer vaccine for postsurgical immunotherapy.

In addition, using peptide hydrogels as delivery vehicles, Yang et al [150] created a vaccine nodule via simultaneously encapsulating exogenous DC cell, OVA antigen, and anti-PD-1 antibody into RADA16 peptide hydrogels (Fig. 10). Peptide RADA16 is an alternating hydrophobic and hydrophilic sequence and has been demonstrated as an efficient hydrogelator to form robust peptide hydrogels [14]. Encapsulation of DC cells within the hydrogels allows for maintaining the cell viability of DC cells, prolonging their duration time at injection site, and facilitating their transportation to lymph nodes. Combination of DC cells and antigens elicits both exogenous and innate DC-associated immune responses, thus amplifying the antigen-specific $\mathrm{T}$ cell immunity. Additional encapsulation of anti-PD-1 antibodies into the hydrogels boosted the proliferation or infiltration of intratumoral $\mathrm{CD}^{+} \mathrm{T}$ cells by preventing the down-regulation of MHC I induced by PD-1/PD-L1 association. While, both in vivo and in vitro experiments confirmed the maturation of DC cells and stimulation of antigenspecific effector $\mathrm{T}$ cells induced by Gel-DC-OVA vaccine; treating mice with Gel-DC-OVA+anti-PD-1 prolonged the survival of tumor-bearing animal and inhibited the growth of tumor significantly. The extraordinary im-
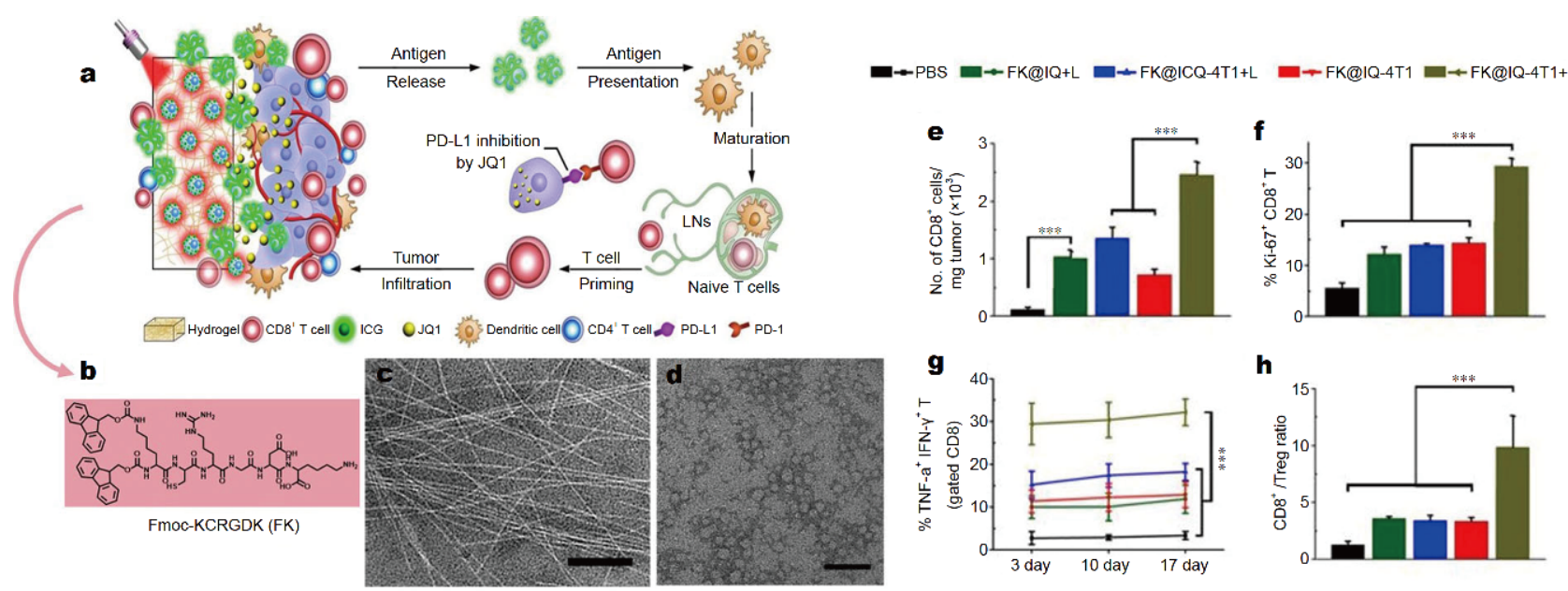

Figure 9 Peptide hydrogels for combinatorial tumor cell antigen and anti-PD-L1 immunotherapy. (a) Schematic representation of the personalized cancer vaccine (PVAX) for postsurgical immunotherapy via simultaneously encapsulating attenuated tumor cells and checkpoint blockades within peptide hydrogels. (b) Chemical structure of Fmoc-KCRGDK (FK) peptide. TEM image of the assemblies of peptide FK after incubation at (c) 37 or (d) $70^{\circ} \mathrm{C}$, respectively. Scale bar: $100 \mathrm{~nm}$ in (c) and $50 \mathrm{~nm}$ in (d). Tumor infiltration (e) and proliferation activity (f) of CD $8^{+} \mathrm{T}$ cells in the recurrent tumors on 10 day after first treatment. (g) Frequency of TNF- $\alpha^{+} / \mathrm{IFN}-\gamma^{+} \mathrm{CD} 8^{+} \mathrm{T}$ cells in the recurrent tumor 3 days after first treatment. (h) Ratios of $\mathrm{CD}^{+} \mathrm{T}$ cells to Tregs in the recurrent tumor 10 days after the first treatment. ${ }^{* *} p<0.01$. Data represent mean \pm s.d. $(n=3)$. Reproduced under the terms of the Creative Commons 4.0 license. [149] Copyright 2018, Nature Publishing Group. 

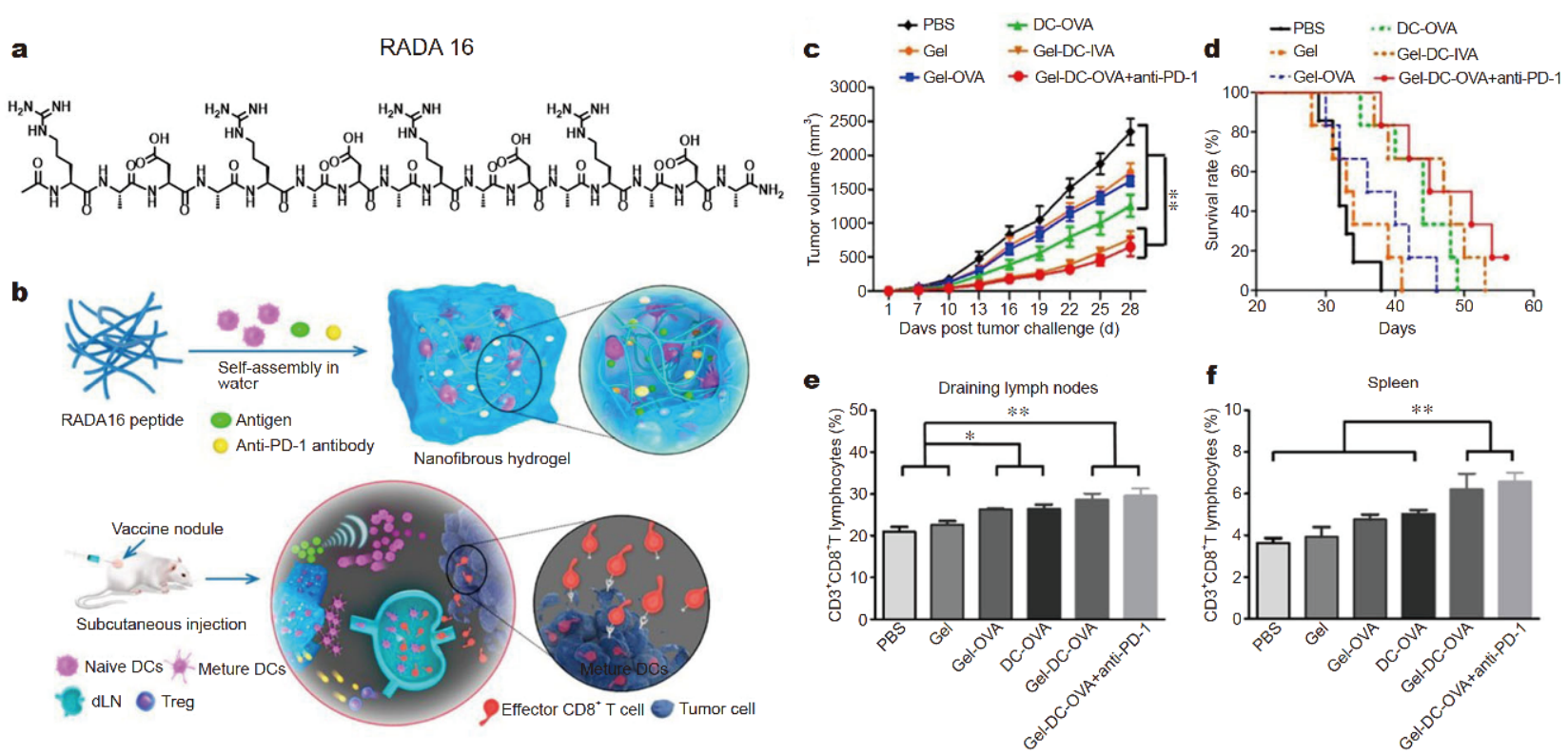

Figure 10 Peptide hydrogels for combinatorial DC-based vaccines and anti-PD-1 immunotherapy. (a) Sequence of peptide RADA. (b) Proposed mechanism of the vaccine nodule composed of RADA hydrogels, encapsulated exogenous DCs, tumor antigen, and anti-PD-1 antibody. (c) Average tumor volumes $(n=5)$ and (d) survival curves $(n=5)$ of mice after treated with vaccine nodule. Day 0 means the first day of tumor inoculation. ${ }^{* *} p<0.01$. (e) The ratios of $\mathrm{CD}^{+} \mathrm{CD}^{+} \mathrm{T}$ cells in the dLNs and (f) in the spleen of vaccinated mice day 28 after tumor challenge $(n=6) .{ }^{*} p<0.05$, ${ }^{* *} p<0.01$. Reproduced with permission from Ref. [150]. Copyright 2018, American Chemical Society.

munogenicity induced by Gel-DC-OVA+anti-PD-1 was attributed to infiltration of $\mathrm{CD} 8^{+} \mathrm{T}$ cell into lymph nodes and suppression of intratumoral Treg cells.

\section{Combinatorial conventional therapy and immunotherapy} In addition to combination of different immune therapeutics, peptide assemblies showed the great potential of co-delivery of therapeutics for both conventional treatments and immunotherapy. For example, both photodynamic therapy and chemotherapy are efficient for inhibiting growth of primary tumors, and also potentially causes immunogenic cell death, which is beneficial for immune responses to enhance antitumor immunity. Therefore, a combination of conventional therapy with immunotherapy exhibits the synergistic therapeutic effect, thus attracting broad attention in preclinical studies. In this context, Song et al [151] reported nanoparticles composed of a chimeric peptide, termed as PpIX-1MT, for combinatorial photodynamic therapy and immunotherapy (Fig. 11). Peptide PpIX-1MT consists of a hydrophobic segment including a palmitic tail and photosensitizer PpIX, and a hydrophilic part including a short PEG chain connecting to an immune checkpoint inhibitor 1-methyltryptophan (1MT) through a caspase3-cleavable DEVD linker. Under the physiological con- dition, peptide PpIX-1MT aggregated into nanoparticles with an average diameter of approximately $128.5 \mathrm{~nm}$ primarily driven by hydrophobic interactions, which targeted tumor cells based on the enhanced permeability and retention effect. Following the apoptosis of cancer cells induced by reactive oxygen species (ROS) produced by photosensitizer PpIX, the induced expression of caspase- 3 cleaved the DEVD sequences and thereby releasing 1MT molecules, which is an IDO inhibitor to prevent the down-regulation of CTL cells and immunosuppression arising from Treg cells. Flow cytometric assay confirmed the immunogenic cell death caused by photodynamic therapy based on the cell-surface exposure of calreticulin, as well as activation of $\mathrm{CD}^{+} \mathrm{T}$ cell immune responses. In vivo studies revealed that combining the cancer cell apoptosis and the immune activation promoted by photodynamic therapy with the enhanced immune response arising from inhibition of IDO allows for eradication of primary tumor and lung metastasis. Insight into the underlying mechanism demonstrated that the inhibition of primary tumor growth was attributed to photodynamic therapy, whereas the activated $\mathrm{CD} 8^{+} \mathrm{T}$ cell immune responses eradicated the lung metastasis, thus establishing a cascaded synergistic therapeutic strategy.

In addition, Zhang's laboratory further developed the 

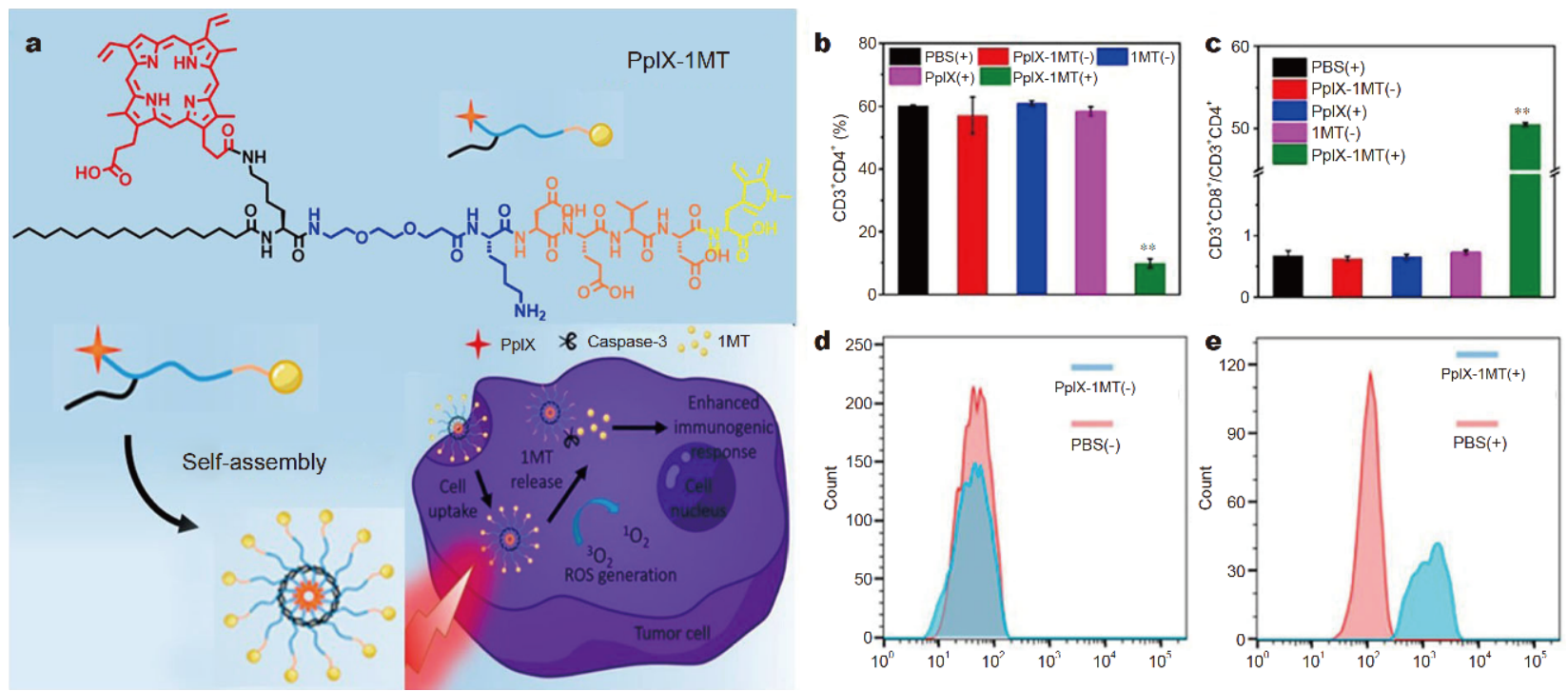

Figure 11 Combinatorial photodynamic therapy and anti-IDO immunotherapy. (a) Chemical structure of peptide PpIX-1MT and schematic illustration of self-assembly of PpIX-1MT into nanoparticles for combinatorial photodynamic therapy and immunotherapy. Ratio of $\mathrm{CD}^{+} \mathrm{T}$ cells to $\mathrm{CD} 3^{+}$ lymphocytes (b) or $\mathrm{CD}^{+} \mathrm{CD}^{+} \mathrm{T}$ cells to $\mathrm{CD}^{+} \mathrm{CD}^{+} \mathrm{T}$ cells (c) in mice immunized in different strategies. Flow cytometry analysis of CRT exposure on the CT26 cell surface after incubation with PBS or PpIX-1MT without (d) or with (e) irradiation. Reproduced with permission from Ref. [151]. Copyright 2018, American Chemical Society.

combinatorial chemotherapy and immunotherapy for treatment of glioblastoma based on simultaneous delivery of chemotherapeutic doxorubicin (DOX) and immune checkpoint inhibitor 1MT into orthotopic glioma (Fig. 12) [152]. The co-delivering system, termed as DOX@MSN-SS-iRGD\&1MT, was composed of mesoporous silica nanoparticles (MSN) loaded with drug DOX and displaying immune checkpoint blockade 1MT and tumor cell targeting epitope iRGD on the surface. While the DOX-loaded MSNs were capped by $\beta$-CDs via disulfide bonds, the surface displaying moieties were noncovalently attached through $\beta$-CD-adamantane association. Connecting inhibitor $1 \mathrm{MT}$ and adamantine via a DEVD domain allows for release of the inhibitor upon exposure to caspase-3, whereas release of DOX from MSNs was promoted by removal of $\beta$-CD caused by reduction of disulfide bonds by GSH. In vitro experiments revealed that treating glioma with DOX@MSN-SSiRGD\&1MT induced apoptosis of glioma cells and elicited antitumor immune responses. In vivo studies demonstrated the capability of DOX@MSN-SS-iRGD\&1MT for penetrating blood brain barrier and spatially delivering and releasing DOX and $1 \mathrm{MT}$ at tumor sites. The synergistic therapeutic of chemotherapy and immunotherapy elicited the CTL immune responses and suppressed the activation of Treg cells, thus eventually leading to prolonged survival of glioma tumor-bearing mice and inhibition of the growth of tumors.

\section{SUMMARY AND OUTLOOK}

Cancer immunotherapy is promising for tumor treatment due to its advantages in eliciting host immune responses to protect against local cancer cells and potentially inducing long-term immune memory to prevent cancer recurrence and metastasis. This review summarized peptide-based strategies for cancer immunotherapy in terms of the therapeutic functions of peptides or peptide assemblies and their mechanism for modulating immune responses. Due to the extraordinary biocompatibility of peptides and their protein-derived structural features, while short peptides have been utilized as therapeutics such as checkpoint blockades, antigens, and vaccine adjuvants, peptide assemblies showed advanced capability in targeting delivery or co-delivery of therapeutics in a controllable manner. Thus far many preclinical studies found the remarkable capability of peptide-based therapeutics for modulation of immune responses and inhibition of tumor growth, demonstrating the great potential of peptide-based immunotherapy in clinical trials.

Despite the progress achieved over the past decade, clinical applications of peptide-based cancer immunotherapy are still challenging and only limited examples have been approved. The primary challenge is the 

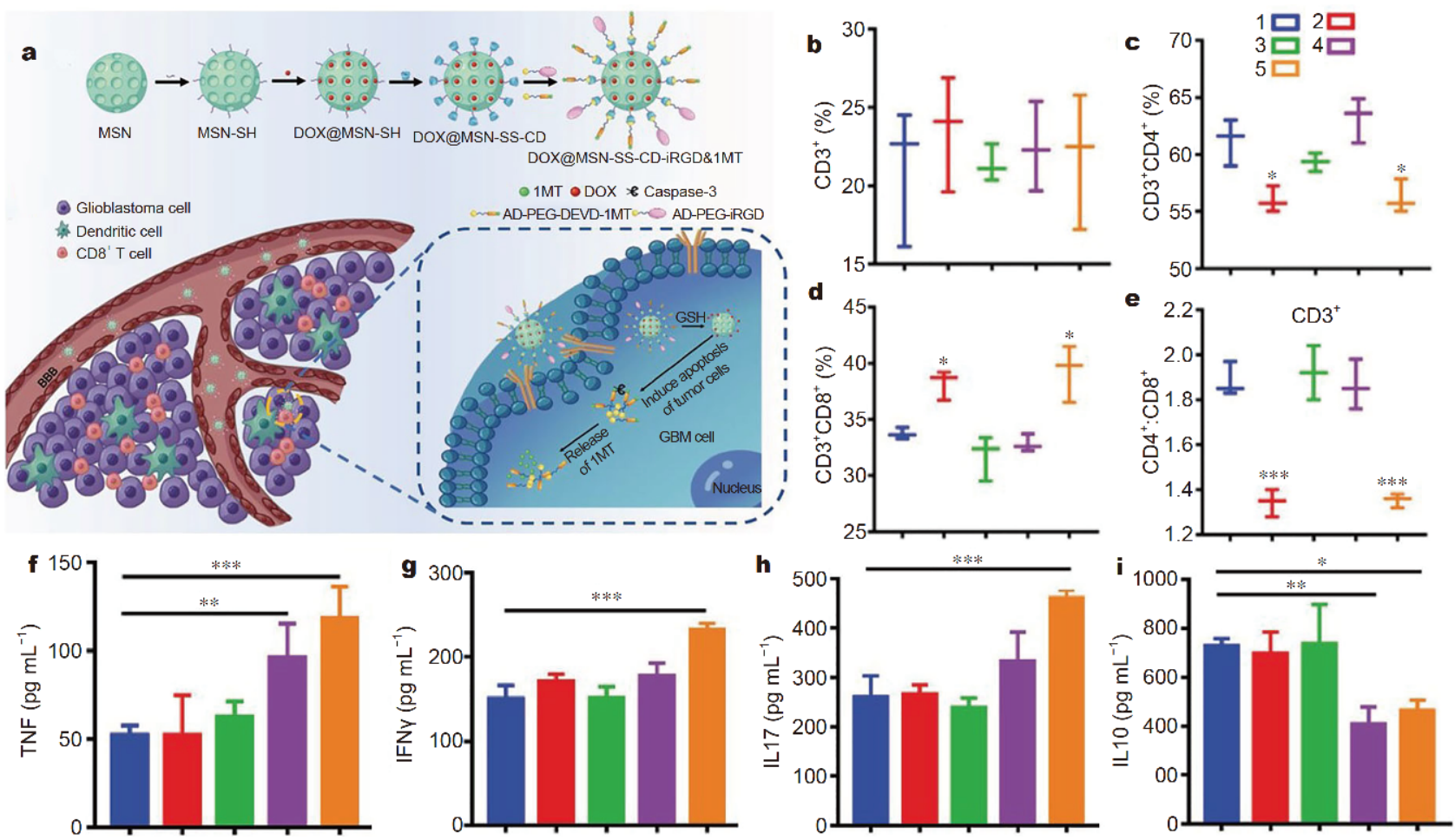

Figure 12 Combinatorial chemotherapy and anti-IDO immunotherapy. (a) Preparation of DOX@MSN-SS-iRGD\&1MT and schematic illustration of DOX@MSN-SS-iRGD\&1MT for eliciting antitumor immunity against glioblastoma and loading DOX for chemotherapy. (b-e) Immune responses induced by DOX@MSN-SS-iRGD\&1MT in vitro: production of $\mathrm{CD}^{+} \mathrm{T}$ cells (b) or cytotoxic $\mathrm{CD} 3^{+} \mathrm{CD} 8^{+} \mathrm{T}$ cells (c) or $\mathrm{CD} 3^{+} \mathrm{CD} 4^{+} \mathrm{T}$ cells $(\mathrm{d})$; (e) Ratio of $\mathrm{CD} 3^{+} \mathrm{CD} 4^{+} \mathrm{T}$ cells to $\mathrm{CD} 3^{+} \mathrm{CD} 8^{+} \mathrm{T}$ cells. ${ }^{*} p<0.05$ and ${ }^{* *} p<0.001$. (f-i) Expression of immune cytokines in orthotopic glioma tissue: (f) TNF; (g) IFN $\gamma$; (h) IL17; and (i) IL10, in brain glioma tissue detected by ELISA. ${ }^{\star} p<0.05$, ${ }^{*} p<0.01$, and ${ }^{* * *} p<0.001$. From (b) to (i), 1: PBS; 2 : free DOX with 1MT; 3: DOX@MSN-SS-CD; 4: DOX@MSN-SS-iRGD; and 5: DOX@MSN-SS-iRGD\&1MT). Reproduced with permission from Ref. [152]. Copyright 2018, John Wiley and Sons.

relative low immunogenicity in most cases, which is attributed to many aspects [153]. Compared to large proteins as antibodies or vaccines, epitopes derived from proteins usually exhibit low selective affinity to specific targets. In addition, this association could be potentially further lowered by the phenotypic heterogeneity of targets or receptors in individuals. Another significant challenge of peptide-based immunotherapy lies in administration safety on the basis of observation of remarkable side effect and syndromes in preclinical trials. These side effect and syndromes could arise from the poor biocompatibility of therapeutics or delivery systems, off-target delivery of therapeutics, and resulting autoimmunity, among others. Although the efficacy of targeting release of therapeutics has been improved by utilizing peptide delivery systems, quantitative release of cargoes at tumor sites remains challenging [154].

Considering the aforementioned challenges, development of therapeutics with high immunogenicity and formulation with acceptable administration safety will be the prospective developing direction of peptide-based immunotherapy. Regarding the development of new therapeutics, design of multivalent peptide checkpoint blockades, antigens, and neontigens [155] is a versatile strategy to improve the affinity of therapeutics with target substrates, thereby potentially leading to high immune responses [156]. The immunogenicity would be potentially improved by creating new delivery systems that enable to increase the infiltration and accumulation of activated $\mathrm{T}$ cells at lymph nodes or integrate multiple immune therapeutics with synergistic effect. In particular, establishment of multi-biomarker-controlled release of drugs and incorporation of multiple target-guiding epitopes into delivery systems likely prevent off-targeting release of therapeutics. In addition, self-assembly of drug amphiphiles based on phase collapse has also been developed as a new strategy for drug delivery, in which few useless species exist in formulations, thus perhaps improving vehicle safety. Combining the thoughts together, peptide-based cancer immunotherapy is a promising 
strategy for cancer treatment in the future.

\section{Received 26 April 2019; accepted 3 June 2019; published online 27 June 2019}

1 Littman DR. Releasing the brakes on cancer immunotherapy. Cell, 2015, 162: 1186-1190

2 Del Paggio JC. Cancer immunotherapy and the value of cure. Nat Rev Clin Oncol, 2018, 15: 268-270

3 Waldmann TA. Immunotherapy: Past, present and future. Nat Med, 2003, 9: 269-277

4 Couzin-Frankel J. Cancer immunotherapy. Science, 2013, 342: 1432-1433

5 Sahin U, Türeci Ö. Personalized vaccines for cancer immunotherapy. Science, 2018, 359: 1355-1360

6 Mellman I, Coukos G, Dranoff G. Cancer immunotherapy comes of age. Nature, 2011, 480: 480-489

7 Byun DJ, Wolchok JD, Rosenberg LM, et al. Cancer immunotherapy-immune checkpoint blockade and associated endocrinopathies. Nat Rev Endocrinol, 2017, 13: 195-207

8 Ribas A, Wolchok JD. Cancer immunotherapy using checkpoint blockade. Science, 2018, 359: 1350-1355

9 Park JH, Geyer MB, Brentjens RJ. CD19-targeted CAR T-cell therapeutics for hematologic malignancies: Interpreting clinical outcomes to date. Blood, 2016, 127: 3312-3320

10 Cheadle EJ, Gornall H, Baldan V, et al. CAR T cells: Driving the road from the laboratory to the clinic. Immunol Rev, 2013, 257: 91-106

11 Cao Y, Rodgers DT, Du J, et al. Design of switchable chimeric antigen receptor $\mathrm{T}$ cells targeting breast cancer. Angew Chem Int Ed, 2016, 55: 7520-7524

12 Golubovskaya V. CAR-T cell therapy: From the bench to the bedside. Cancers, 2017, 9: 150

13 Brudno JN, Kochenderfer JN. Chimeric antigen receptor T-cell therapies for lymphoma. Nat Rev Clin Oncol, 2018, 15: 31-46

14 Zhang S. Discovery and design of self-assembling peptides. Interface Focus, 2017, 7: 20170028

15 Cui H, Webber MJ, Stupp SI. Self-assembly of peptide amphiphiles: From molecules to nanostructures to biomaterials. Biopolymers, 2010, 94: 1-18

16 Barnes CP, Sell SA, Boland ED, et al. Nanofiber technology: Designing the next generation of tissue engineering scaffolds. Adv Drug Deliver Rev, 2007, 59: 1413-1433

17 Zasloff M. Antimicrobial peptides of multicellular organisms. Nature, 2002, 415: 389-395

18 Mahlapuu M, Håkansson J, Ringstad L, et al. Antimicrobial peptides: An emerging category of therapeutic agents. Front Cell Infect Microbiol, 2016, 6

19 Lee S, Xie J, Chen X. Peptides and peptide hormones for molecular imaging and disease diagnosis. Chem Rev, 2010, 110: 30873111

20 Abbas M, Zou Q, Li S, et al. Self-assembled peptide- and proteinbased nanomaterials for antitumor photodynamic and photothermal therapy. Adv Mater, 2017, 29: 1605021

$21 \mathrm{Li} \mathrm{J}, \mathrm{Pu} \mathrm{K}$. Development of organic semiconducting materials for deep-tissue optical imaging, phototherapy and photoactivation. Chem Soc Rev, 2019, 48: 38-71

22 Miao Q, Yeo DC, Wiraja C, et al. Near-infrared fluorescent molecular probe for sensitive imaging of keloid. Angew Chem Int Ed, 2018, 57: 1256-1260
23 Yin $\mathrm{L}$, Sun $\mathrm{H}$, Zhang $\mathrm{H}$, et al. Quantitatively visualizing tumorrelated protease activity in vivo using a ratiometric photoacoustic probe. J Am Chem Soc, 2019, 141: 3265-3273

24 Zhang P, Cheetham AG, Lin YA, et al. Self-assembled tat nanofibers as effective drug carrier and transporter. ACS Nano, 2013, 7: 5965-5977

25 Zhang N, Zhao F, Zou Q, et al. Multitriggered tumor-responsive drug delivery vehicles based on protein and polypeptide coassembly for enhanced photodynamic tumor ablation. Small, 2016, 12: $5936-5943$

26 Agemy L, Friedmann-Morvinski D, Ramana Kotamraju V, et al. Targeted nanoparticle enhanced proapoptotic peptide as potential therapy for glioblastoma. Proc Natl Acad Sci USA, 2011, 108: 17450-17455

27 Junttila MR, de Sauvage FJ. Influence of tumour micro-environment heterogeneity on therapeutic response. Nature, 2013, 501: 346-354

28 Liu Y, Zhang D, Qiao ZY, et al. A peptide-network weaved nanoplatform with tumor microenvironment responsiveness and deep tissue penetration capability for cancer therapy. Adv Mater, 2015, 27: 5034-5042

29 Gupta B, Levchenko TS, Torchilin VP. Intracellular delivery of large molecules and small particles by cell-penetrating proteins and peptides. Adv Drug Deliver Rev, 2005, 57: 637-651

30 Curnis F, Sacchi A, Borgna L, et al. Enhancement of tumor necrosis factor a antitumor immunotherapeutic properties by targeted delivery to aminopeptidase N (CD13). Nat Biotechnol, 2000, 18: 1185-1190

31 Hoshino Y, Kodama T, Okahata Y, et al. Peptide imprinted polymer nanoparticles: A plastic antibody. J Am Chem Soc, 2008, 130: $15242-15243$

32 Rudra JS, Mishra S, Chong AS, et al. Self-assembled peptide nanofibers raising durable antibody responses against a malaria epitope. Biomaterials, 2012, 33: 6476-6484

33 Cieslewicz M, Tang J, Yu JL, et al. Targeted delivery of proapoptotic peptides to tumor-associated macrophages improves survival. Proc Natl Acad Sci USA, 2013, 110: 15919-15924

34 Genové E, Shen C, Zhang S, et al. The effect of functionalized selfassembling peptide scaffolds on human aortic endothelial cell function. Biomaterials, 2005, 26: 3341-3351

35 Pompano RR, Chen J, Verbus EA, et al. Titrating T-cell epitopes within self-assembled vaccines optimizes $\mathrm{CD}^{+}$helper $\mathrm{T}$ cell and antibody outputs. Adv Healthcare Mater, 2014, 3: 1898-1908

36 Luo L, Zhu C, Yin H, et al. Laser immunotherapy in combination with perdurable PD-1 blocking for the treatment of metastatic tumors. ACS Nano, 2018, 12: 7647-7662

37 Peng J, Yang Q, Xiao Y, et al. Tumor microenvironment responsive drug-dye-peptide nanoassembly for enhanced tumortargeting, penetration, and photo-chemo-immunotherapy. Adv Funct Mater, 2019, 29: 1900004

38 Kuai R, Ochyl LJ, Bahjat KS, et al. Designer vaccine nanodiscs for personalized cancer immunotherapy. Nat Mater, 2016, 16: 489496

39 Kakwere H, Ingham ES, Allen R, et al. Toward personalized peptide-based cancer nanovaccines: A facile and versatile synthetic approach. Bioconjugate Chem, 2017, 28: 2756-2771

40 Chen J, Pompano RR, Santiago FW, et al. The use of self-adjuvanting nanofiber vaccines to elicit high-affinity $\mathrm{B}$ cell responses to peptide antigens without inflammation. Biomaterials, 2013, 34: 8776-8785 
41 Koutsopoulos S, Unsworth LD, Nagai Y, et al. Controlled release of functional proteins through designer self-assembling peptide nanofiber hydrogel scaffold. Proc Natl Acad Sci USA, 2009, 106: 4623-4628

42 Li Y, Wang F, Cui H. Peptide-based supramolecular hydrogels for delivery of biologics. Bioeng Translat Med, 2016, 1: 306-322

43 Koutsopoulos S, Zhang S. Two-layered injectable self-assembling peptide scaffold hydrogels for long-term sustained release of human antibodies. J Control Release, 2012, 160: 451-458

44 Liu R, Yu M, Yang X, et al. Linear chimeric triblock molecules self-assembled micelles with controllably transformable property to enhance tumor retention for chemo-photodynamic therapy of breast cancer. Adv Funct Mater, 2019, 29: 1808462

45 Jin P, Sha R, Zhang Y, et al. Blood circulation-prolonging peptides for engineered nanoparticles identified via phage display. Nano Lett, 2019, 19: 1467-1478

$46 \mathrm{Ng} \mathrm{CW}, \mathrm{Li} \mathrm{J}, \mathrm{Pu}$ K. Recent progresses in phototherapy-synergized cancer immunotherapy. Adv Funct Mater, 2018, 28: 1804688

47 Wang D, Wang $\mathrm{T}$, Liu J, et al. Acid-activatable versatile micelleplexes for PD-L1 blockade-enhanced cancer photodynamic immunotherapy. Nano Lett, 2016, 16: 5503-5513

48 He C, Duan X, Guo N, et al. Core-shell nanoscale coordination polymers combine chemotherapy and photodynamic therapy to potentiate checkpoint blockade cancer immunotherapy. Nat Commun, 2016, 7: 12499

$49 \mathrm{Xu} \mathrm{J}$, Xu L, Wang C, et al. Near-infrared-triggered photodynamic therapy with multitasking upconversion nanoparticles in combination with checkpoint blockade for immunotherapy of colorectal cancer. ACS Nano, 2017, 11: 4463-4474

50 Yu S, Wang C, Yu J, et al. Injectable bioresponsive gel depot for enhanced immune checkpoint blockade. Adv Mater, 2018, 30: 1801527

51 Buchbinder EI, Desai A. CTLA-4 and PD-1 pathways. Am J Clin Oncol, 2016, 39: 98-106

52 Sharma P, Allison JP. The future of immune checkpoint therapy. Science, 2015, 348: 56-61

53 Gubin MM, Zhang X, Schuster $\mathrm{H}$, et al. Checkpoint blockade cancer immunotherapy targets tumour-specific mutant antigens. Nature, 2014, 515: 577-581

54 Pardoll DM. The blockade of immune checkpoints in cancer immunotherapy. Nat Rev Cancer, 2012, 12: 252-264

55 Parry RV, Chemnitz JM, Frauwirth KA, et al. CTLA-4 and PD-1 receptors inhibit T-cell activation by distinct mechanisms. Mol Cellular Biol, 2005, 25: 9543-9553

56 Schwartz RH. Costimulation of t lymphocytes: The role of CD28, CTLA-4, and B7/BB1 in interleukin-2 production and immunotherapy. Cell, 1992, 71: 1065-1068

57 Triebel F, Jitsukawa S, Baixeras E, et al. LAG-3, a novel lymphocyte activation gene closely related to CD4. J Exp Med, 1990, 171: 1393-1405

58 Huang CT, Workman CJ, Flies D, et al. Role of LAG-3 in regulatory T cells. Immunity, 2004, 21: 503-513

59 Grosso JF, Kelleher CC, Harris TJ, et al. LAG-3 regulates $\mathrm{CD}^{+}{ }^{+} \mathrm{T}$ cell accumulation and effector function in murine self- and tumor-tolerance systems. J Clin Invest, 2007, 117: 3383-3392

60 Monney L, Sabatos CA, Gaglia JL, et al. Th1-specific cell surface protein TIM-3 regulates macrophage activation and severity of an autoimmune disease. Nature, 2002, 415: 536-541

61 Zhu C, Anderson AC, Schubart A, et al. The TIM-3 ligand galectin-9 negatively regulates $t$ helper type 1 immunity. Nat Im- munol, 2005, 6: 1245-1252

62 Fourcade J, Sun Z, Benallaoua M, et al. Upregulation of Tim-3 and $\mathrm{PD}-1$ expression is associated with tumor antigen-specific $\mathrm{CD}^{+} \mathrm{T}$ cell dysfunction in melanoma patients. J Exp Med, 2010, 207: 2175-2186

63 Fife BT, Bluestone JA. Control of peripheral T-cell tolerance and autoimmunity via the CTLA-4 and PD-1 pathways. Immunological Rev, 2010, 224: 166-182

$64 \mathrm{Yu}$ X, Harden K, C Gonzalez L, et al. The surface protein TIGIT suppresses $\mathrm{T}$ cell activation by promoting the generation of mature immunoregulatory dendritic cells. Nat Immunol, 2008, 10: 48-57

65 Joller N, Lozano E, Burkett PR, et al. Treg cells expressing the coinhibitory molecule TIGIT selectively inhibit proinflammatory Th1 and Th17 cell responses. Immunity, 2014, 40: 569-581

66 Walker LSK, Sansom DM. The emerging role of CTLA4 as a cellextrinsic regulator of $\mathrm{T}$ cell responses. Nat Rev Immunol, 2011, 11: 852-863

67 Walunas TL, Lenschow DJ, Bakker CY, et al. CTLA-4 can function as a negative regulator of $\mathrm{T}$ cell activation. Immunity, 1994, 1: $405-413$

68 Leach DR, Krummel MF, Allison JP. Enhancement of antitumor immunity by CTLA-4 blockade. Science, 1996, 271: 1734-1736

69 Peggs KS, Quezada SA, Chambers CA, et al. Blockade of CTLA-4 on both effector and regulatory $\mathrm{T}$ cell compartments contributes to the antitumor activity of anti-CTLA-4 antibodies. J Exp Med, 2009, 206: 1717-1725

70 Krummel MF, Allison JP. CD28 and CTLA-4 have opposing effects on the response of T cells to stimulation. J Exp Med, 1995, 182: $459-465$

71 Wing K, Onishi Y, Prieto-Martin P, et al. CTLA-4 control over Foxp $3^{+}$regulatory T cell function. Science, 2008, 322: 271-275

72 Zou W, Wolchok JD, Chen L. PD-L1 (B7-H1) and PD-1 pathway blockade for cancer therapy: Mechanisms, response biomarkers, and combinations. Sci Transl Med, 2016, 8: 328rv4

73 Francisco LM, Sage PT, Sharpe AH. The PD-1 pathway in tolerance and autoimmunity. Immunological Rev, 2010, 236: 219242

74 Latchman Y, Wood CR, Chernova T, et al. PD-L2 is a second ligand for PD-1 and inhibits T cell activation. Nat Immunol, 2001, 2: 261-268

75 Chen L. Co-inhibitory molecules of the B7-CD28 family in the control of T-cell immunity. Nat Rev Immunol, 2004, 4: 336-347

76 Tumeh PC, Harview CL, Yearley JH, et al. PD-1 blockade induces responses by inhibiting adaptive immune resistance. Nature, 2014, 515: 568-571

77 Gordon SR, Maute RL, Dulken BW, et al. PD-1 expression by tumour-associated macrophages inhibits phagocytosis and tumour immunity. Nature, 2017, 545: 495-499

78 Boutros C, Tarhini A, Routier E, et al. Safety profiles of antiCTLA-4 and anti-PD-1 antibodies alone and in combination. Nat Rev Clin Oncol, 2016, 13: 473-486

79 Reilly RM, Sandhu J, Alvarez-Diez TM, et al. Problems of delivery of monoclonal antibodies. Clin Pharm, 1995, 28: 126-142

80 Chen T, Li Q, Liu Z, et al. Peptide-based and small synthetic molecule inhibitors on PD-1/PD-L1 pathway: A new choice for immunotherapy? Eur J Medicinal Chem, 2019, 161: 378-398

81 Chang HN, Liu BY, Qi YK, et al. Blocking of the PD-1/PD-L1 interaction by a D-peptide antagonist for cancer immunotherapy. Angew Chem Int Ed, 2015, 54: 11760-11764 
82 Maute RL, Gordon SR, Mayer AT, et al. Engineering high-affinity PD-1 variants for optimized immunotherapy and immuno-pet imaging. Proc Natl Acad Sci USA, 2015, 112: E6506-E6514

83 Li C, Zhang N, Zhou J, et al. Peptide blocking of PD-1/PD-L1 interaction for cancer immunotherapy. Cancer Immunol Res, 2018, 6: 178-188

84 Munir Ahmad S, Martinenaite E, Hansen M, et al. PD-L1 peptide co-stimulation increases immunogenicity of a dendritic cell-based cancer vaccine. Oncoimmunology, 2016, 5: e1202391

85 Magiera-Mularz K, Skalniak L, Zak KM, et al. Bioactive macrocyclic inhibitors of the PD-1/PD-L1 immune checkpoint. Angew Chem Int Ed, 2017, 56: 13732-13735

86 Molino NM, Anderson AKL, Nelson EL, et al. Biomimetic protein nanoparticles facilitate enhanced dendritic cell activation and cross-presentation. ACS Nano, 2013, 7: 9743-9752

87 Black M, Trent A, Kostenko Y, et al. Self-assembled peptide amphiphile micelles containing a cytotoxic T-cell epitope promote a protective immune response in vivo. Adv Mater, 2012, 24: 3845-3849

88 Qian Y, Jin H, Qiao S, et al. Targeting dendritic cells in lymph node with an antigen peptide-based nanovaccine for cancer immunotherapy. Biomaterials, 2016, 98: 171-183

89 Jäger E, Chen YT, Drijfhout JW, et al. Simultaneous humoral and cellular immune response against cancer-testis antigen NY-ESO1: definition of human histocompatibility leukocyte antigen (HLA)-A2-binding peptide epitopes. J Exp Med, 1998, 187: 265270

90 Neek M, Tucker JA, Kim TI, et al. Co-delivery of human cancertestis antigens with adjuvant in protein nanoparticles induces higher cell-mediated immune responses. Biomaterials, 2018, 156: 194-203

91 Jäeger E, Bernhard H, Romero P, et al. Generation of cytotoxic Tcell responses with synthetic melanoma-associated peptides in vivo: Implications for tumor vaccines with melanoma-associated antigens. Int J Cancer, 1996, 66: 162-169

92 Jäger E, Höhn $\mathrm{H}$, Necker A, et al. Peptide-specific $\mathrm{CD} 8^{+} \mathrm{T}$-cell evolution in vivo: Response to peptide vaccination with Melan-A/ MART-1. Int J Cancer, 2002, 98: 376-388

93 Slingluff. CLJ, Yamshchikov G, Neese P, et al. Phase I trial of a melanoma vaccine with gp100(280-288) peptide and tetanus helper peptide in adjuvant: Immunologic and clinical outcomes. Clin Cancer Res, 2001, 7: 3012-3024

94 Tarhini AA, Leng S, Moschos SJ, et al. Safety and immunogenicity of vaccination with MART-1 (26-35, 27L), gp100 (209-217, $210 \mathrm{M})$, and tyrosinase $(368-376,370 \mathrm{D})$ in adjuvant with PF3512676 and GM-CSF in metastatic melanoma. J ImmunoTher, 2012, 35: 359-366

95 Guo Y, Wang D, Song Q, et al. Erythrocyte membrane-enveloped polymeric nanoparticles as nanovaccine for induction of antitumor immunity against melanoma. ACS Nano, 2015, 9: 69186933

96 Wakabayashi R, Sakuragi M, Kozaka S, et al. Solid-in-oil peptide nanocarriers for transcutaneous cancer vaccine delivery against melanoma. Mol Pharm, 2018, 15: 955-961

97 Haugen A. Cancer, genes and gender. Carcinogenesis, 2002, 23: 1089

98 Peoples GE, Holmes JP, Hueman MT, et al. Combined clinical trial results of a HER2/neu (E75) vaccine for the prevention of recurrence in high-risk breast cancer patients: U.S. military cancer institute clinical trials group study I-01 and I-02. Clinical
Cancer Res, 2008, 14: 797-803

99 Villa CH, Dao T, Ahearn I, et al. Single-walled carbon nanotubes deliver peptide antigen into dendritic cells and enhance IgG responses to tumor-associated antigens. ACS Nano, 2011, 5: 53005311

100 Ressing ME, Sette A, Brandt RMP, et al. Human CTL epitopes encoded by human papillomavirus type 16 E6 and E7 identified through in vivo and in vitro immunogenicity studies of HLA$A^{\star} 0201$-binding peptides. J Immunol, 1995, 154: 5934-5943

101 van Driel WJ, Ressing ME, Kenter GG, et al. Vaccination with HPV16 peptides of patients with advanced cervical carcinoma: clinical evaluation of a phase I-II trial. Eur J Cancer, 1999, 35: 946-952

102 Liu TY, Hussein WM, Jia Z, et al. Self-adjuvanting polymerpeptide conjugates as therapeutic vaccine candidates against cervical cancer. Biomacromolecules, 2013, 14: 2798-2806

103 Liu TY, Hussein WM, Giddam AK, et al. Polyacrylate-based delivery system for self-adjuvanting anticancer peptide vaccine. J Med Chem, 2015, 58: 888-896

104 Tindle RW, Croft S, Herd K, et al. A vaccine conjugate of 'ISCAR' immunocarrier and peptide epitopes of the E7 cervical cancerassociated protein of human papillomavirus type 16 elicits specific Th1- and Th2-type responses in immunized mice in the absence of oil-based adjuvants. Clin Exp Immunol, 1995, 101: 265-271

105 Tsoras AN, Champion JA. Cross-linked peptide nanoclusters for delivery of oncofetal antigen as a cancer vaccine. Bioconj Chem, 2018, 29: 776-785

106 Rudra JS, Tian YF, Jung JP, et al. A self-assembling peptide acting as an immune adjuvant. Proc Natl Acad Sci USA, 2010, 107: 622627

107 Rudra JS, Sun T, Bird KC, et al. Modulating adaptive immune responses to peptide self-assemblies. ACS Nano, 2012, 6: 15571564

108 Luo Z, Wu Q, Yang C, et al. A powerful CD8 ${ }^{+} \mathrm{T}$-cell stimulating D-tetra-peptide hydrogel as a very promising vaccine adjuvant. Adv Mater, 2017, 29: 1601776

109 Yang C, Shi F, Li C, et al. Single dose of protein vaccine with peptide nanofibers as adjuvants elicits long-lasting antibody titer. ACS Biomater Sci Eng, 2017, 4: 2000-2006

110 Wang Z, Liang C, Shi F, et al. Cancer vaccines using supramolecular hydrogels of NSAID-modified peptides as adjuvants abolish tumorigenesis. Nanoscale, 2017, 9: 14058-14064

111 Melero I, Gaudernack G, Gerritsen W, et al. Therapeutic vaccines for cancer: An overview of clinical trials. Nat Rev Clin Oncol, 2014, 11: 509-524

112 van Duin D, Medzhitov R, Shaw AC. Triggering TLR signaling in vaccination. Trends Immunol, 2006, 27: 49-55

113 Parkin J, Cohen B. An overview of the immune system. Lancet, 2001, 357: 1777-1789

114 Zheng Y, Wen Y, George AM, et al. A peptide-based material platform for displaying antibodies to engage T cells. Biomaterials, 2011, 32: 249-257

115 König R, Huang LY, Germain RN. MHC class II interaction with CD4 mediated by a region analogous to the MHC class I binding site for CD8. Nature, 1992, 356: 796-798

116 Plotkin SA. Vaccines: Past, present and future. Nat Med, 2005, 11: S5-S11

117 Matsumura M, Fremont DH, Peterson PA, et al. Emerging principles for the recognition of peptide antigens by MHC class I 
molecules. Science, 1992, 257: 927-934

118 Skwarczynski M, Toth I. Recent advances in peptide-based subunit nanovaccines. Nanomedicine, 2014, 9: 2657-2669

119 Zhu G, Zhang F, Ni Q, et al. Efficient nanovaccine delivery in cancer immunotherapy. ACS Nano, 2017, 11: 2387-2392

120 Rosenberg SA, Yang JC, Schwartzentruber DJ, et al. Immunologic and therapeutic evaluation of a synthetic peptide vaccine for the treatment of patients with metastatic melanoma. Nat Med, 1998, 4: $321-327$

121 Bakker AB, Schreurs MW, de Boer AJ, et al. Melanocyte lineagespecific antigen gp100 is recognized by melanoma- derived tumor-infiltrating lymphocytes. J Exp Med, 1994, 179: 1005-1009

122 Speiser $\mathrm{D}$, Liénard $\mathrm{D}$, Pittet $\mathrm{M}$, et al. In vivo activation of melanoma-specific $\mathrm{CD}^{+} \mathrm{T}$ cells by endogenous tumor antigen and peptide vaccines. A comparison to virus-specific T cells. Eur J Immunol, 2002, 32: 731

123 Skakuj K, Wang S, Qin L, et al. Conjugation chemistry-dependent T-cell activation with spherical nucleic acids. J Am Chem Soc, 2018, 140: 1227-1230

124 Xie J, Yang C, Liu Q, et al. Encapsulation of hydrophilic and hydrophobic peptides into hollow mesoporous silica nanoparticles for enhancement of antitumor immune response. Small, 2017, 13: 1701741

125 Tao Y, Zhang Y, Ju E, et al. Gold nanocluster-based vaccines for dual-delivery of antigens and immunostimulatory oligonucleotides. Nanoscale, 2015, 7: 12419-12426

126 Gendler SJ. MUC1, the renaissance molecule. J Mammary Gland Biol Neoplasia, 2001, 6: 339-353

127 Huang ZH, Shi L, Ma JW, et al. A totally synthetic, self-assembling, adjuvant-free MUC1 glycopeptide vaccine for cancer therapy. J Am Chem Soc, 2012, 134: 8730-8733

128 Liu Y, Wang Y, Yu F, et al. Potentiating the immune response of MUC1-based antitumor vaccines using a peptide-based nanovector as a promising vaccine adjuvant. Chem Commun, 2017, 53: $9486-9489$

129 de Faria PCB, dos Santos LI, Coelho JP, et al. Oxidized multiwalled carbon nanotubes as antigen delivery system to promote superior $\mathrm{CD}^{+} \mathrm{T}$ cell response and protection against cancer. Nano Lett, 2014, 14: 5458-5470

130 Rad-Malekshahi M, Fransen MF, Krawczyk M, et al. Self-assembling peptide epitopes as novel platform for anticancer vaccination. Mol Pharm, 2017, 14: 1482-1493

131 Feltkamp MCW, Vreugdenhil GR, Vierboom MPM, et al. Cytotoxic T lymphocytes raised against a subdominant epitope offered as a synthetic peptide eradicate human papillomavirus type 16induced tumors. Eur J Immunol, 1995, 25: 2638-2642

132 Acar H, Srivastava S, Chung EJ, et al. Self-assembling peptidebased building blocks in medical applications. Adv Drug Deliver Rev, 2017, 110-111: 65-79

133 Reed SG, Orr MT, Fox CB. Key roles of adjuvants in modern vaccines. Nat Med, 2013, 19: 1597-1608

134 Appavu R, Chesson CB, Koyfman AY, et al. Enhancing the magnitude of antibody responses through biomaterial stereochemistry. ACS Biomater Sci Eng, 2015, 1: 601-609

135 Wang H, Luo Z, Wang Y, et al. Enzyme-catalyzed formation of supramolecular hydrogels as promising vaccine adjuvants. Adv Funct Mater, 2016, 26: 1822-1829

136 Gazit E. Self-assembled peptide nanostructures: The design of molecular building blocks and their technological utilization.
Chem Soc Rev, 2007, 36: 1263-1269

137 Hendricks MP, Sato K, Palmer LC, et al. Supramolecular assembly of peptide amphiphiles. Acc Chem Res, 2017, 50: 24402448

138 Cui H, Muraoka T, Cheetham AG, et al. Self-assembly of giant peptide nanobelts. Nano Lett, 2009, 9: 945-951

139 Hamley IW. Peptide fibrillization. Angew Chem Int Ed, 2007, 46: 8128-8147

140 De Santis E, Ryadnov MG. Peptide self-assembly for nanomaterials: The old new kid on the block. Chem Soc Rev, 2015, 44: $8288-8300$

$141 \mathrm{Hu} \mathrm{X}, \mathrm{Hu}$ J, Tian J, et al. Polyprodrug amphiphiles: Hierarchical assemblies for shape-regulated cellular internalization, trafficking, and drug delivery. J Am Chem Soc, 2013, 135: 17617-17629

142 Hudalla GA, Sun T, Gasiorowski JZ, et al. Gradated assembly of multiple proteins into supramolecular nanomaterials. Nat Mater, 2014, 13: 829-836

143 Demaria S, Kawashima N, Yang AM, et al. Immune-mediated inhibition of metastases after treatment with local radiation and CTLA- 4 blockade in a mouse model of breast cancer. Clin Cancer Res, 2005, 11: 728-734

144 Sharabi AB, Lim M, DeWeese TL, et al. Radiation and checkpoint blockade immunotherapy: Radiosensitisation and potential mechanisms of synergy. Lancet Oncol, 2015, 16: e498-e509

145 Fan W, Yung B, Huang P, et al. Nanotechnology for multimodal synergistic cancer therapy. Chem Rev, 2017, 117: 13566-13638

146 Sharma P, Allison JP. Immune checkpoint targeting in cancer therapy: Toward combination strategies with curative potential. Cell, 2015, 161: 205-214

147 Mahoney KM, Rennert PD, Freeman GJ. Combination cancer immunotherapy and new immunomodulatory targets. Nat Rev Drug Discov, 2015, 14: 561-584

148 Cheng K, Ding Y, Zhao Y, et al. Sequentially responsive therapeutic peptide assembling nanoparticles for dual-targeted cancer immunotherapy. Nano Lett, 2018, 18: 3250-3258

149 Wang $\mathrm{T}$, Wang $\mathrm{D}, \mathrm{Yu} \mathrm{H}$, et al. A cancer vaccine-mediated postoperative immunotherapy for recurrent and metastatic tumors. Nat Commun, 2018, 9: 1532

150 Yang P, Song H, Qin Y, et al. Engineering dendritic-cell-based vaccines and $\mathrm{PD}-1$ blockade in self-assembled peptide nanofibrous hydrogel to amplify antitumor T-cell immunity. Nano Lett, 2018, 18: 4377-4385

151 Song W, Kuang J, Li CX, et al. Enhanced immunotherapy based on photodynamic therapy for both primary and lung metastasis tumor eradication. ACS Nano, 2018, 12: 1978-1989

152 Kuang J, Song W, Yin J, et al. iRGD modified chemo-immunotherapeutic nanoparticles for enhanced immunotherapy against glioblastoma. Adv Funct Mater, 2018, 28: 1800025

153 Skwarczynski M, Toth I. Peptide-based synthetic vaccines. Chem Sci, 2016, 7: 842-854

154 Parmiani G, Russo V, Maccalli C, et al. Peptide-based vaccines for cancer therapy. Human Vaccines Immunotherapeutics, 2014, 10: 3175-3178

155 Zacco E, Anish C, Martin CE, et al. A self-assembling peptide scaffold for the multivalent presentation of antigens. Biomacromolecules, 2015, 16: 2188-2197

156 Eskandari S, Guerin T, Toth I, et al. Recent advances in self-assembled peptides: Implications for targeted drug delivery and vaccine engineering. Adv Drug Deliver Rev, 2017, 110-111: 169-187 
Acknowledgements This work was supported by the National Natural Science Foundation of China (21774065), the Fundamental Research Funds for the Central Universities, and the Natural Science Foundation of Tianjin (18JCQNJC14100).

Author contributions $\mathrm{Li} \mathrm{M}$ prepared the manuscript under the guidance of Yu Z. Li M and Zhao X designed and prepared the figures. $\mathrm{Yu} \mathrm{Z}$ and Dai J revised the manuscript. All authors contributed to the general discussion and revision of the manuscript.

Conflict of interest The authors declare no conflict of interest.

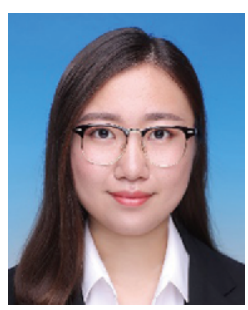

Mingming Li received her Master degree in chemical engineering from China University of Petroleum (Beijing) in 2018. Currently she is a $\mathrm{PhD}$ candidate under the supervision of Prof. Zhilin Yu at the Institute of Polymer Chemistry of Nankai University. Her current research interest lies in the field of stimulus-responsive peptide self-assembly and the biomaterials including drug delivery and disease therapy.

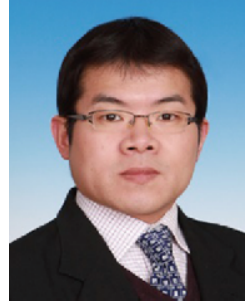

Zhilin Yu was awarded his $\mathrm{PhD}$ degree under the supervision of Prof. Stefan Hecht at the Humboldt-Universität zu Berlin in 2013. He conducted his postdoctoral training with Prof. Samuel I. Stupp at Northwestern University focusing on self-assembly of peptide-based amphiphilic molecules. In 2017, he started his independent career at the Institute of Polymer Science of Nankai University. His current research interests focus on the self-assembly of peptides into dynamic nanostructures and their broad applications as biomaterials including disease diagnosis and therapy.

\section{多肽药物及组装体在癌症免疫治疗中的应用}

李明明, 赵欣然, 戴建芳, 余志林 ${ }^{*}$

摘要 基于通过激活宿主天然免疫应答杀死癌症细胞的优势, 免疫 治疗有望成为癌症治疗的新方法. 与传统治疗方法相比, 免疫治疗 能够诱导长期的免疫记忆以预防癌症复发和转移, 具有更广谱的 抗癌效果以及较小的副作用. 然而建立具有高生物安全性和免疫 应答能力的策略仍然具有挑战性. 由于其与天然蛋白质类似的结 构特征, 多肽分子有望通过直接引发免疫应答或改善药物递送效 果来解决这些挑战. 本文总结了过去十年内发现的利用短肽分子 作为免疫治疗药物或递送平台的癌症免疫疗法. 从简要介绍癌症 免疫治疗开始, 我们概述了多肽分子的特定药物功能, 包括免疫检 查点抑制剂、疫苗抗原和佐剂. 随后着重介绍了基于多肽纳米结 构作为递送平台, 用于药物靶向递送或多种药物共同递送以增强 免疫原性的进展. 最后对基于多肽的癌症免疫治疗面临的挑战以 及未来的发展趋势进行了展望. 